\title{
The Spatial Characteristics of Reactive Stepping Among People Living with Chronic Incomplete Spinal Cord Injury
}

by

Matthew G. Heffernan

A thesis submitted in conformity with the requirements

for the degree of Master of Science

Rehabilitation Sciences

University of Toronto

(C) Copyright by Matthew G. Heffernan 2021 


\title{
The Spatial Characteristics of Reactive Stepping Among People Living with Chronic Incomplete Spinal Cord Injury
}

\author{
Matthew G. Heffernan \\ Master of Science \\ Rehabilitation Sciences \\ University of Toronto
}

2021

\begin{abstract}
People with incomplete spinal cord injuries (iSCI) are more likely to require multiple reactive steps to maintain balance after a simulated forward fall when compared with able-bodied (AB) individuals. To better understand these behavioural differences, we aimed to compare the length and width of the first reactive step between people with chronic motor iSCI and AB individuals. Participants attended testing sessions where the spatial characteristics of a reactive step initiated using the Lean-and-Release test were examined. The magnitude of step length and width did not differ between groups, but participants with iSCI demonstrated significantly greater variability in the length and width of their first reactive step. Additionally, we assessed the spatial measurement properties of the Lean-and-Release test and found it was a reliable and valid measure of mean step length for people with iSCI. Altogether, the findings suggest targets for reactive balance assessment and training for people living with iSCI.
\end{abstract}




\section{Acknowledgments}

I would like to thank Dr. Kristin Musselman for supporting me through the last two years. I was privileged to have her guidance as I completed my degree while navigating the unforeseen challenges of a global pandemic. I have greatly appreciated the learning environment she has fostered at the SCI Mobility Lab and look forward to continuing my studies under her supervision.

Thanks to my fellow SCI Mobility Lab members for their support and friendship. I have learned a great deal from all of them and their research excellence inspires me. I would like to offer my special thanks to, Jae Lee, Katherine Chan, and Dr. Janelle Unger. It was a privilege to continue with the work they started and I strived to match the quality of their research. The helpful advice and assistance they offered along the way were greatly appreciated.

I was first introduced to SCI research when Dr. Kei Masani offered me a summer position in his lab as an undergraduate student and I thank him for his continued encouragement and guidance. Thank you also to Dr. Susan Marzolini and Dr. Tim Welsh for their supervision. The knowledge they offered to me has improved my research skills and the quality of my projects.

Finally, I would like to thank my family for always encouraging me to pursue my dreams. They motivate me to do my best and I would not be where I am today without their love and support. 


\section{Table of Contents}

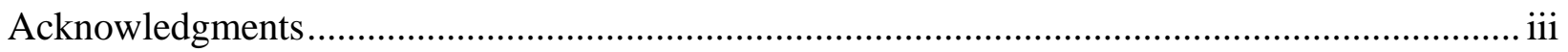

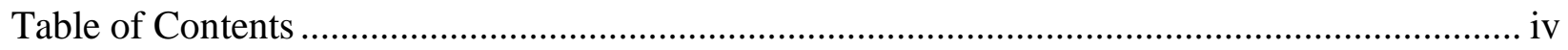

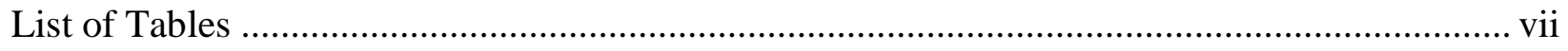

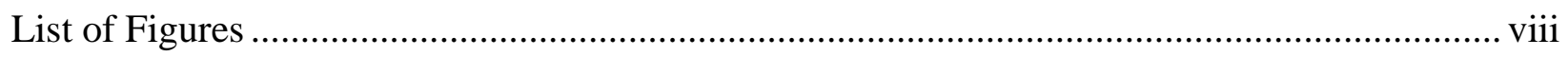

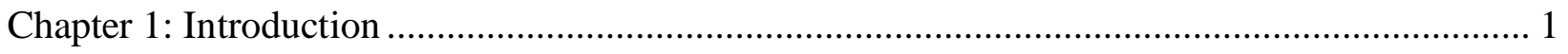

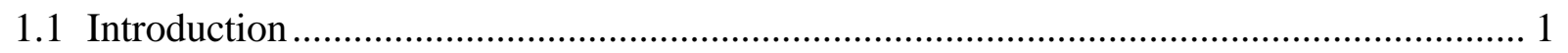

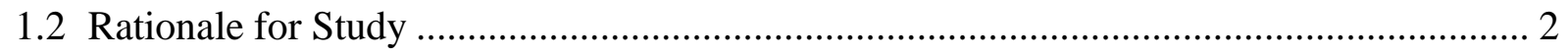

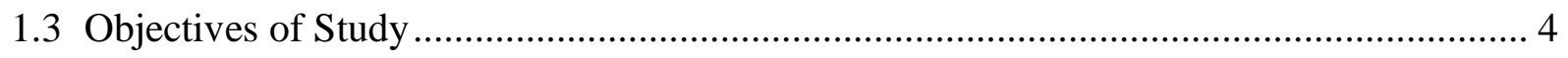

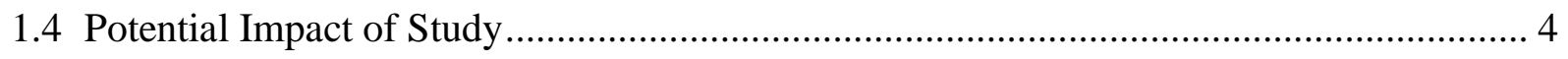

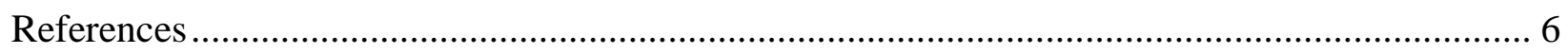

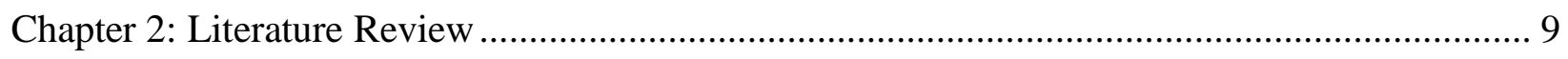

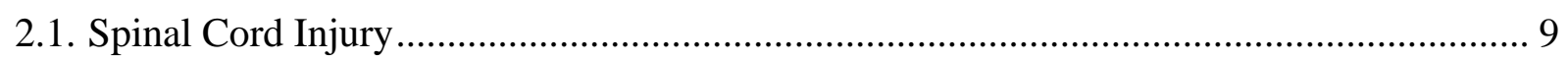

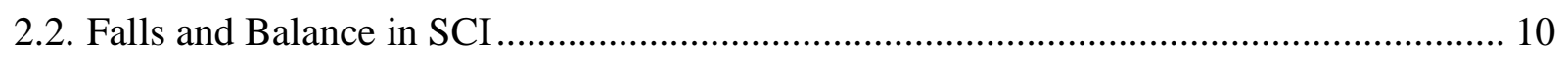

2.2.1. Postural Control in SCI ............................................................................... 10

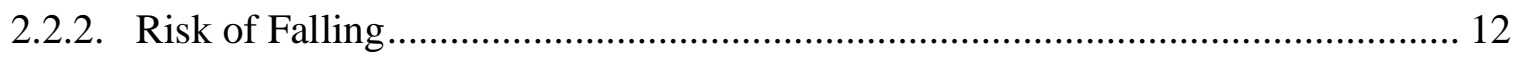

2.2.3. Reactive Balance and iSCI.................................................................. 12

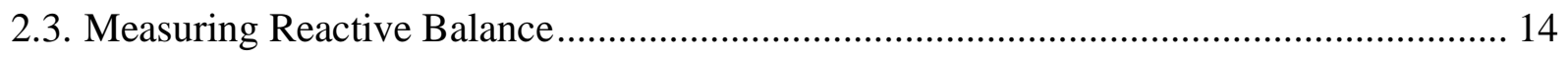

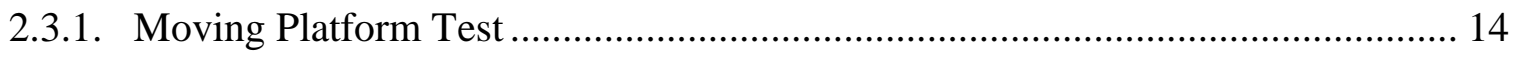

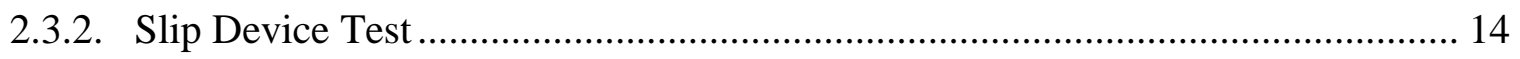

2.3.3. Lean-and-Release Test ......................................................................... 15

2.3.4. Psychometric Properties of the Lean-and-Release Test.................................... 16

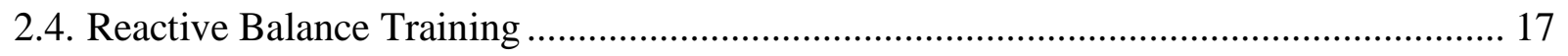


Chapter 3: Spatial Characteristics of Reactive Stepping Among People Living with Chronic Incomplete Spinal Cord Injury ............................................................................... 28

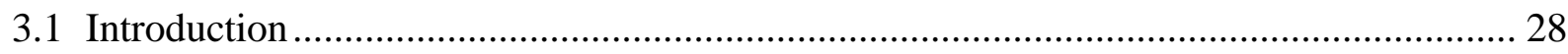

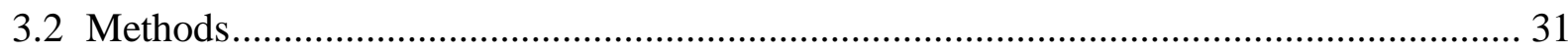

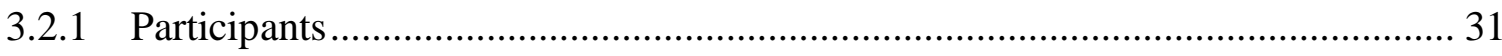

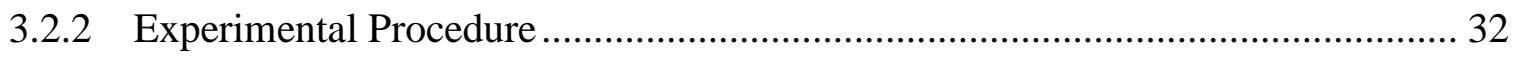

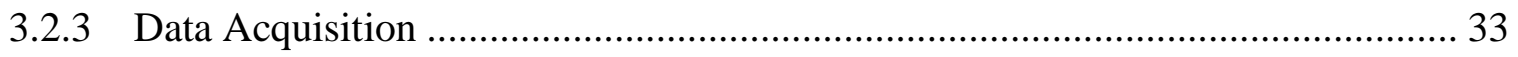

3.2.4 Data Analysis .................................................................................... 35

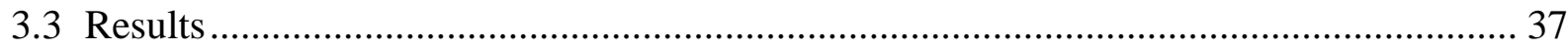

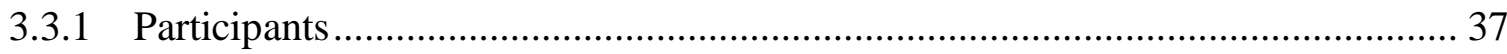

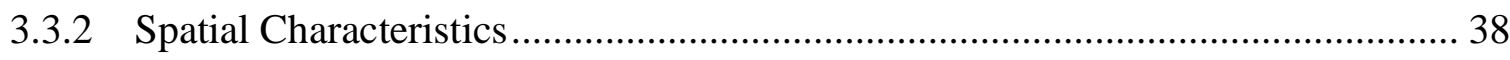

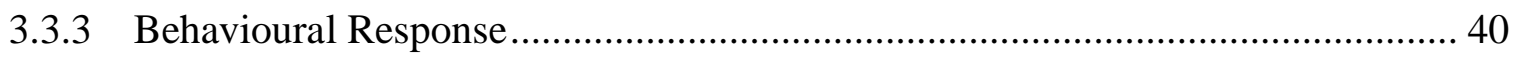

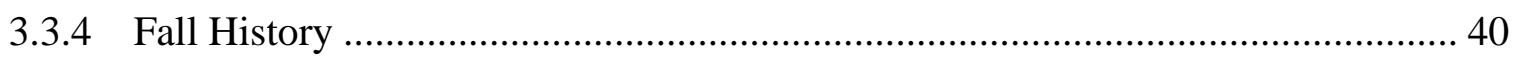

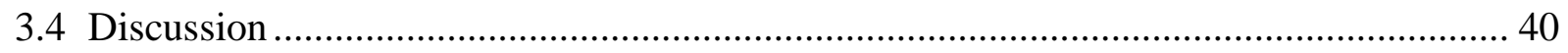

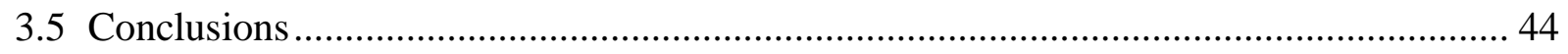

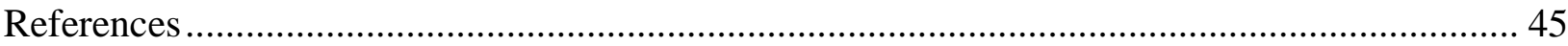

Chapter 4: Evaluating the Spatial Measurement Properties of the Lean-and-Release Test for Individuals with Incomplete Spinal Cord Injuries ......................................................... 51

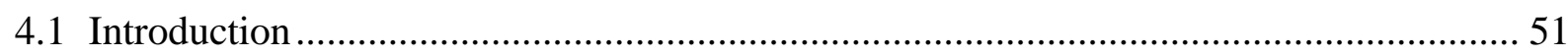

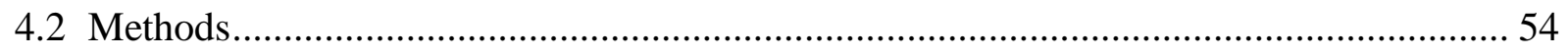

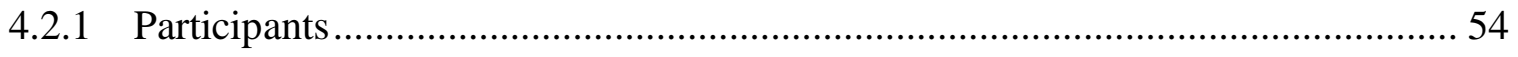

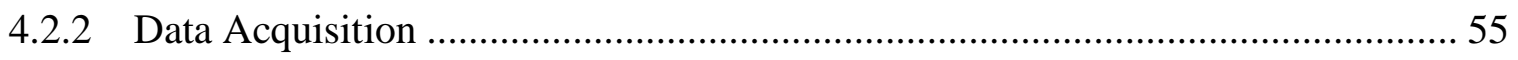

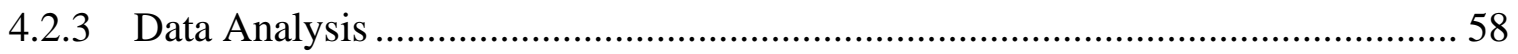

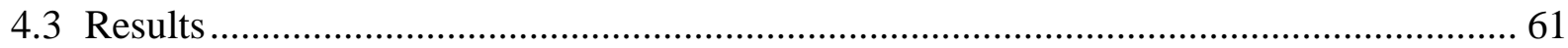

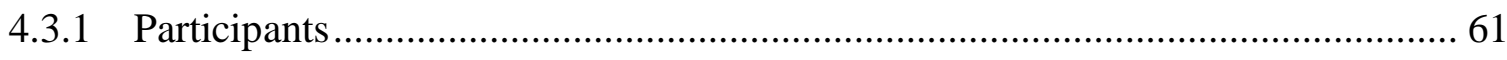

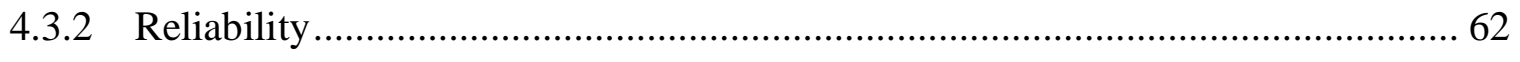




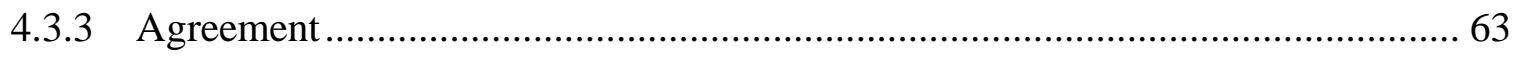

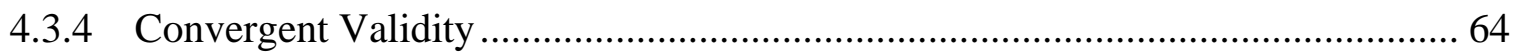

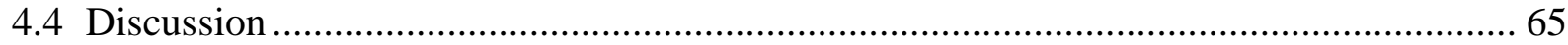

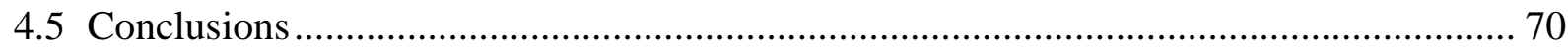

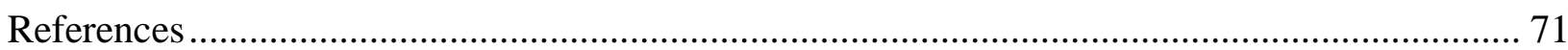

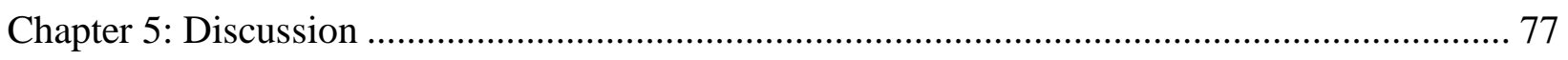

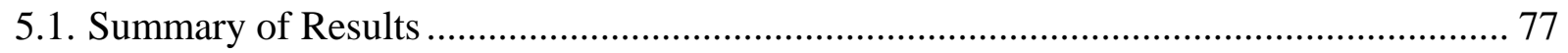

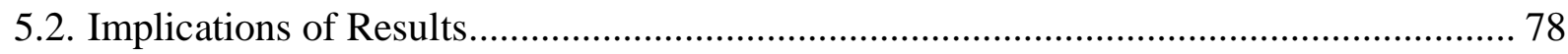

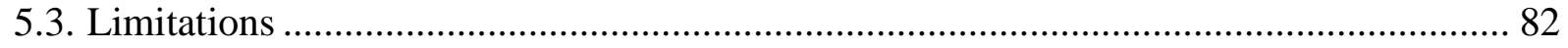

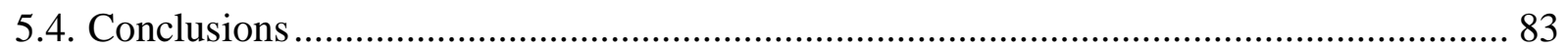

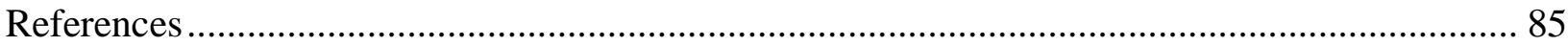

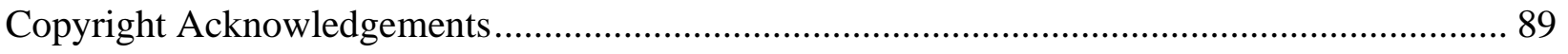




\section{List of Tables}

Table 3.1. Demographic, injury and fall history of iSCI participants......................... 38

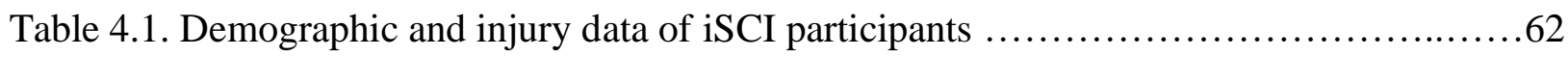

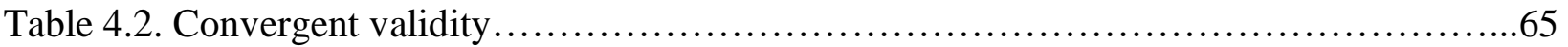




\section{List of Figures}

Figure 3.1. Configuration of the Lean-and-Release test.............................33, 58

Figure 3.2. Measurement of spatial characteristics.................................... 36,61

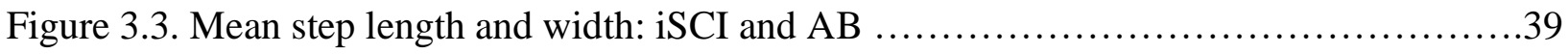

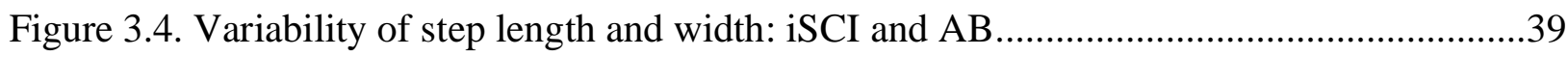

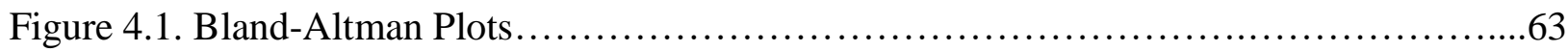




\section{Chapter 1}

\section{Introduction}

\subsection{Introduction}

For ambulatory individuals with incomplete spinal cord injury (iSCI), reactive stepping ability is important for avoiding falls and subsequent fall-related conditions which often have lasting negative impacts on one's life (Brotherton et al., 2007; Maki \& Mcilroy, 2006; Musselman et al., 2018; Singh et al., 2020). Behavioural differences in reactive stepping have been observed between able-bodied (AB) individuals and people with iSCI, with the latter being more likely to require multiple steps after a simulated forward fall (Chan et al., 2020). Using a cross-sectional design, this study examined potential contributors to these behavioural differences. Specifically, this study aimed to compare the spatial characteristics of the first reactive step between people with iSCI and AB individuals. Reactive stepping was assessed using the Lean-and-Release test, a measurement tool that simulates a fall from a forward leaning position (Inness et al., 2015). Participants were subjected to multiple Lean-and-Release trials in which the spatial characteristics of their stepping response after a forward fall were recorded using motion capture and force plate systems that quantified change in position of the heel of a stepping foot. The secondary aim of this study was to assess the measurement properties of the spatial characteristics of reactive stepping as measured with the Lean-and-Release test for people with iSCI. Participants completed two testing sessions, the data from which were used to determine the test-retest reliability, agreement, and construct validity of the Lean-and-Release test's spatial parameters (i.e., step length and width). The results of this study suggest the length and width of the first reactive steps taken by individual's with iSCI are similar in magnitude, but more 
variable, compared to $\mathrm{AB}$ individuals. Additionally, as a measure of mean step length, the Leanand-Release test was found to have good test-retest reliability and demonstrated a high degree of convergent validity based on correlations with other balance and gait related measures.

\subsection{Rationale for Study}

Each year, $78 \%$ of ambulatory people with iSCI experience at least one fall and nearly $50 \%$ experience three or more falls (Jørgensen et al., 2017; Khan et al., 2019). These falls are often dangerous events that cause injury requiring urgent medical care and the consequences can be long lasting as many develop a fear of falling that can limit an individual's independence and quality of life (Brotherton et al., 2007; Musselman et al., 2018; Singh et al., 2020). As a result, fall prevention is an important focus of rehabilitation and training after SCI. One strategy used to prevent falls after an unexpected perturbation is a reactive stepping response, which serves to increase one's base of support to account for the rapid change in position of one's centre of mass (Hilliard et al., 2008; Maki \& Mcilroy, 2006; Mansfield et al., 2012; Mansfield et al., 2013; McCrum et al., 2017). To effectively rehabilitate and train reactive stepping in people with iSCI, it is important to understand which mechanisms of this response may be deficient.

A recent study by Chan et al. (2020), found that after a simulated forward fall, people with iSCI were more likely to take multiple reactive steps compared to $\mathrm{AB}$ individuals. This suggests that the first reactive step taken by individuals with iSCI may not be adequate for maintaining balance. Despite this observed difference in the behavioural response, contributing deficiencies in the underlying mechanisms of reactive stepping remain uncertain. For example, individuals with iSCI have demonstrated similar movement timing (i.e. foot off and foot contact time) during the first reactive step compared to AB individuals (Chan et al., 2020). Additionally, six leg 
muscles were examined using surface electromyography and although the onset of muscle activation was found to be more variable in individuals with iSCI, only the onset of activation in the tibialis anterior muscle of the stepping leg was consistently delayed compared to $\mathrm{AB}$ individuals (Chan et al., 2020). In other populations, it has been suggested that deficiencies in the spatial characteristics of a reactive step may contribute to a diminished reactive stepping ability. For example, older $\mathrm{AB}$ adults who have an impaired reactive stepping ability have been shown to take shorter reactive step lengths when compared to younger AB adults (Hsiao-Wecksler \& Robinovitch, 2007; Medell \& Alexander, 2000). However, the spatial characteristics of the first reactive step have not yet been examined for people with iSCI, representing an important opportunity for further study.

The Lean-and-Release test simulates a forward fall and can be used to measure many parameters of reactive stepping (Inness et al., 2015). The test involves a participant, secured by a safety harness, leaning forward while a horizontal cable attached to their back at waist height supports them. A forward fall is initiated once the cable is released, and a reactive stepping response is required in order to maintain balance. During the test, behavioural (i.e., the number of steps taken), temporal (i.e., foot off, foot contact, and swing time) and spatial (i.e., step length and width) data can be recorded through integrated force plate and motion capture technologies. As the focus on reactive stepping has grown, use of the Lean-and-Release test as an assessment tool for clinical populations has also increased (Chan et al., 2020; Inness et al., 2015; Mansfield et al., 2011). As a result of its growing use, recent studies have set out to assess the psychometric properties of the Lean-and-Release test. One study in particular examined the reliability and validity of the behavioural and temporal parameters of the Lean-and-Release test for individuals with iSCI (Unger et al., 2020). The behavioural response of the test was found to be reliable and valid for people with iSCI although just one of the temporal parameters (foot contact) 
demonstrated good test-retest reliability (Unger et al., 2020). Currently, the reliability and validity of the Lean-and-Release test for measuring spatial parameters of reactive stepping in people with iSCI have not been established.

\subsection{Objectives of Study}

Objective 1: To compare the spatial characteristics of reactive stepping after a simulated forward fall between individuals with iSCI and AB individuals.

Objective 2: To evaluate the spatial measurement properties of the lean-and-release test for individuals with iSCI.

\subsection{Potential Impact of Study}

This study compared the spatial characteristics of reactive stepping during the Lean-and-Release test between people with iSCI and AB individuals. The results suggest that the mean length and width of the first reactive step taken by individuals with iSCI are similar in magnitude, but more variable, when compared to AB individuals. Further, the Lean-and-Release test was found to be reliable and valid for measuring mean step length. Recently, clinical research has tested balance training interventions for people with stroke (Mansfield et al., 2018) and iSCI (Unger et al., 2021). These studies have used the Lean-and-Release test to assess the effectiveness of the intervention. Future research investigating the effect of balance training interventions for people with iSCI can utilize the results presented in this study by directing focus to certain spatial aspects of the reactive step. For example, based on our results, a balance training intervention may be considered effective if it reduces the variability of one's first reactive step length and width. Additionally, this study demonstrated that the Lean-and-Release test is a reliable and valid 
measure of mean reactive step length for people with iSCI and therefore, examination of this parameter is warranted when evaluating reactive stepping ability. 


\section{References}

Brotherton, S. S., Krause, J. S., \& Nietert, P. J. (2007). Falls in individuals with incomplete spinal cord injury. Spinal Cord. 45(1), 37-40.

Chan, K., Lee, J. W., Unger, J., Yoo, J., Masani, K., \& Musselman, K. E. (2020). Reactive stepping after a forward fall in people living with incomplete spinal cord injury or disease. Spinal Cord. 58, 185-193.

Hilliard, M. J., Martinez, K. M., Janssen, I., Edwards, B., Mille, M.-L., Zhang, Y., \& Rogers, M. W. (2008). Lateral balance factors predict future falls in community-living older adults. Archives of Physical Medicine and Rehabilitation, 89(9), 1708-1713.

Hsiao-Wecksler, E. T., \& Robinovitch, S. N. (2007). The effect of step length on young and elderly women's ability to recover balance. Clinical Biomechanics, 22(5), 574-580.

Inness, E. L., Mansfield, A., Biasin, L., Brunton, K., Bayley, M., \& McIlroy, W. E. (2015). Clinical implementation of a reactive balance control assessment in a sub-acute stroke patient population using a 'lean-and-release' methodology. Gait Posture. 41(2), 529-34.

Jørgensen, V., Butler Forslund, E., Opheim, A., Franzén, E., Wahman, K., Hultling, C., Seiger, Å., Ståhle, A., Stanghelle, J. K., \& Roaldsen, K. S. (2017). Falls and fear of falling predict future falls and related injuries in ambulatory individuals with spinal cord injury: a longitudinal observational study. Journal of Physiotherapy, 63(2), 108-113.

Khan, A., Pujol, C., Laylor, M., Unic, N., Pakosh, M., Dawe, J., \& Musselman, K. E. (2019). Falls after spinal cord injury: A systematic review and meta-analysis of incidence proportion and contributing factors. Spinal Cord. 57(7), 526-539. 
Maki, B. E., \& Mcilroy, W. E. (2006). Control of rapid limb movements for balance recovery: Age-related changes and implications for fall prevention. Age Ageing, 35(2), ii12-ii18.

Mansfield, A., Aqui, A., Danells, C. J., Knorr, S., Centen, A., DePaul, V. G., Schinkel-Ivy, A., Brooks, D., Inness, E. L., \& Mochizuki, G. (2018). Does perturbation-based balance training prevent falls among individuals with chronic stroke? A randomised controlled trial. BMJ Open, 8(8), e021510.

Mansfield, A., Inness, E. L., Komar, J., Biasin, L., Brunton, K., Lakhani, B., \& McIlroy, W. E. (2011). Training rapid stepping responses in an individual with stroke. Physical Therapy, 91(6), 958-969.

Mansfield, A., Inness, E. L., Lakhani, B., \& McIlroy, W. E. (2012). Determinants of limb preference for initiating compensatory stepping post- stroke. Archives of Physical Medicine and Rehabilitation, 93(7), 1179-1184.

Mansfield, A., Inness, E. L., Wong, J. S., Fraser, J. E., \& McIlroy, W. E. (2013). Is impaired control of reactive stepping related to falls during inpatient stroke rehabilitation? Neurorehabilitation and Neural Repair, 27(6), 526-533.

McCrum, C., Gerards, M. H. G., Karamanidis, K., Zijlstra, W., \& Meijer, K. (2017). A systematic review of gait perturbation paradigms for improving reactive stepping responses and falls risk among healthy older adults. European Review of Aging and Physical Activity, 14(1). 
Medell, J. L., \& Alexander, N. B. (2000). A clinical measure of maximal and rapid stepping in older women. The Journals of Gerontology Series A: Biological Sciences and Medical Sciences, 55(8), M429-M433.

Musselman, K. E., Arnold, C., Pujol, C., Lynd, K., \& Oosman, S. (2018). Falls, mobility and physical activity after spinal cord injury: An exploratory study using photo-elicitation interviewing. Spinal Cord Series and Cases. 4, 39.

Singh, H., Shibi Rosen, A., Bostick, G., Kaiser, A., \& Musselman, K. E. (2020). Exploring the causes and impacts of falls among ambulators with spinal cord injury using photovoice: A mixed methods study. BMJ Open. 10, e039763.

Unger, J., Chan, K., Lee, J. W., Craven, B. C., Mansfield, A., Alavinia, M., Masani, K., \& Musselman, K. E. (2021). The effect of Perturbation-based Balance Training and Conventional Intensive Balance Training on reactive stepping ability in individuals with incomplete spinal cord injury or disease: a randomized clinical trial. Frontiers in Neurology, 12.

Unger, J., Oates, A. R., Lanovaz, J., Chan, K., Lee, J. W., Theventhiran, P., Masani, K., \& Musselman, K. E. (2020). The measurement properties of the Lean-and-Release test in people with incomplete spinal cord injury or disease. The Journal of Spinal Cord Medicine, $1-10$. 


\section{Chapter 2}

\section{Literature Review}

\subsection{Spinal Cord Injury}

A spinal cord injury (SCI) occurs as a result of traumatic (i.e. falls and motor vehicle accidents) or non-traumatic (i.e., infection or disease) damage to the spinal cord, which disrupts sensorimotor communication between the spinal cord and the brain (Wierbecky \& Kamath, 2013). Typically, information from the motor cortex of the brain travels through descending efferent fibres to the spinal cord and from the spinal cord through ascending afferent fibres to the brain (Hamid \& Hayek, 2008). However, when the spinal cord is damaged this line of communication is disrupted and although the integrity of spinal circuits below the level of injury may remain unharmed, the amount of descending input from the brain which can reach those circuits is often insufficient (Hamid \& Hayek, 2008). As a result, muscle contractions requiring neural input below the level the injury are impaired causing paralysis and decreased independence in functional abilities (Doucet et al., 2012). As defined by the American Spinal Injury Association Impairment Scale (AIS), the degree of these sensorimotor impairments can range from complete loss of sensory and motor function below the level of the injury (AIS level A SCI) to incomplete injuries characterized by some preservation of sensory and motor function (AIS level C and D SCI).

Globally, traumatic SCI are estimated to effect 10.5 people per 100,000 (Kumar et al. 2018) and non-traumatic SCI, although more difficult to identify, are estimated to effect 36.7 people per 100,000 (New et al., 2013; New et al., 2017). Although the global prevalence of SCI is relatively 
low compared to other conditions, the associated healthcare costs are significant. For example, in Canada, the lifetime economic cost of a spinal cord injury can range from 1.5 to 3 million dollars depending on the severity of the injury (Krueger et al. 2013). For people with SCI, the impact on one's daily life is substantial. The sensory, motor, and autonomic dysfunction caused by SCI often leads to significant decreases in an individual's functional independence and mobility, which negatively impacts quality of life (Musselman et al., 2019).

\subsection{Falls and Balance in SCI}

\subsubsection{Postural Control in SCI}

One of the primary aims of rehabilitation following SCI is to maximize independence through the retraining of different movements that facilitate the safe completion of activities of daily living. Achieving adequate postural control, or balance, during these movements is critical to the goal of regaining independence (Musselman et al., 2021). Postural control and balance are linked concepts that are often used synonymously to describe one's ability to avoid falls. Specifically, balance is defined by the position of one's centre of mass (CoM) relative to their base of support (BoS) (Maki \&McIlroy, 2006) and postural control refers to one's ability to attain a state balance by using corrective muscle contractions to reposition one's CoM such that it remains or returns within their BoS (Pollock et al., 2000). Depending on the condition (i.e. standing or walking) different systems of postural control may be involved in maintaining balance (Horak, 2006). For example, during standing, an individual's biomechanical constraints (i.e. limits of stability, strength, and coordination) and orientation (i.e. perception of verticality and gravity) are critical for maintaining balance (Horak, 2006; Sibley et al., 2015). When an individual is walking however, their dynamic stability (i.e. ability to control CoM as BoS changes) is heavily relied 
upon (Horak, 2006; Sibley et al., 2015). Other unique movements also require specific systems of postural control. For example, anticipatory postural control can be used to change the position of one's CoM before a movement occurs and reactive postural control refers to an individual's ability to reposition CoM within BoS after an external perturbation (Horak, 2006; Sibley et al., 2015).

The spinal cord plays an important role in producing the muscle contractions that regulate postural control. Specifically, descending motor pathways in the spinal cord activate postural synergies (Beloozerova et al. 2005; Zelenin et al. 2010) and ascending somatosensory pathways to the brain facilitate environmental adaptation (Chiba et al. 2016). For individuals with SCI, a disruption in the flow of information below the level of injury impairs the ability to make muscle contractions and as a result, many systems of postural control are negatively affected (Musselman et al., 2021). During standing for example, individuals with SCI have been shown to have decreased limits of functional stability (Houston et al., 2020; Lemay et al., 2014), decreased movement coordination (Noamani et al., 2020), and increased postural sway (Lemay et al. 2013). Additionally, during walking, dynamic stability may be impaired after SCI as evidenced by increased gait variability (Day et al. 2011; Lemay et al., 2020). Finally, although anticipatory postural control is likely to be minimally affected in people with less severe SCI (Bone et al., 2021; Thigpen et al., 2009), reactive postural control has been shown to be significantly impaired as people with SCI are more likely to require multiple steps to maintain balance after a forward fall (Chan et al., 2020) and have a decreased ability to increase margin of stability (i.e. the distance between margins of one's BoS and their extrapolated CoM) after a slip (Arora et al. 2020; Hof et al., 2005). 


\subsubsection{Risk of Falling}

As a result of impaired postural control, falling is a serious issue for people with incomplete spinal cord injuries (iSCI) with previous research demonstrating that $78 \%$ of these individuals experience one or more falls annually (Khan et al., 2019). These falls often occur during walking with approximately $50 \%$ of ambulatory individuals reporting recurring falls (Jørgensen et al., 2017). Often falls among individuals with iSCI have immediate and serious medical consequences carrying significant associated healthcare costs (Brotherton et al., 2007). Additionally, falls and fall related injuries likely result in substantial secondary consequences for people with iSCI. Specifically, the behaviour of people with iSCI often changes following a fall. For example, many people develop a fear of falling which leads to increased levels of depression and decreases in daily mobility, autonomy, and quality of life (Jørgensen \& Roaldsen, 2017; Musselman et al., 2018; Singh et al., 2021*, Singh et al. 2020)

\subsubsection{Reactive Balance and iSCI}

One specific situation that requires adequate postural control in order to prevent a fall is an unexpected perturbation like a trip or push (Pollock et al. 2000). Unlike predictable perturbations that require sufficient anticipatory balance strategies (i.e., walking slowly over ice), unexpected perturbations require effective reactive balance responses, which occur more rapidly (Maki \& McIlroy, 1997). One rapid response used to avoid a fall after an unexpected perturbation is a quick reactive step (Maki \& McIlroy, 2006). This response works to quickly adjust the position of an individual's CoM such that it remains within their base of support (Hilliard et al., 2008; Mansfield et al., 2012; Mansfield et al., 2013; McCrum et al., 2017). A reactive step is thought to involve two phases. First, the preparation phase is characterized by the reaction time after the 
unexpected perturbation followed by the execution phase that is characterized by the temporal and spatial parameters of the step (Do et al., 1982). If the first reactive step does not sufficiently compensate for the initial change in CoM position, individuals will often take an additional step to maintain balance (Hilliard et al., 2008; Mansfield et al., 2012; Mansfield et al., 2013; McCrum et al., 2017).

Although the results of one study suggest that people with iSCI are able to maintain balance after unpredictable surface perturbations requiring corrective postural sway (Thigpen et al., 2009), other studies have demonstrated impaired reactive balance in response to larger perturbations. For individuals with iSCI, the difficulty of maintaining balance after a perturbation causing a forward fall may be exacerbated by an impaired reactive stepping ability. A recent study comparing the reactive stepping ability of people with iSCI and able-bodied (AB) individuals, demonstrated an increased likelihood to require multiple reactive steps after a simulated forward fall for people with iSCI (Chan et al., 2020). This study also demonstrated increased variability in the onset of muscle activation for individuals with iSCI however, no significant differences were found in the temporal parameters (i.e., movement timing) of the reactive step between the two groups (Chan et al., 2020). Another study found that, compared with AB individuals, people with iSCI have a decreased ability to increase margin of stability, specifically in the lateral direction (i.e., step width is insufficient) after a slip while walking (Arora et al. 2020).

In other groups with impaired reactive balance like people living with stroke, the foot clearance of reactive steps is often insufficient for preventing falls (Mansfield et al., 2012). In Older AB adults, a shorter reactive step in response to a forward fall has been observed (Hageman \& Blanke, 1986; Hsiao-Wecksler \& Robinovitch, 2007; Medell \& Alexander, 2000; Oberg et al., 
1993). However, differences in the spatial characteristics (i.e., step length and step width) have not yet been examined in individuals with iSCI.

\subsection{Measuring Reactive Balance}

\subsubsection{Moving Platform Test}

One method that has been used to evaluate reactive balance in people with iSCI involves participants standing on a platform that moves unpredictably with a backward translation or forward rotation (Thigpen et al., 2009). These horizontal movements of the platform are controlled by a hydraulic system and require body sway in the anterior-posterior direction to maintain balance (Thigpen et al., 2009). During the test, participants experienced five alternating blocks of at least six backward translations or forward rotations that were randomly delayed from trial to trial, reducing anticipatory responses (Thigpen et al., 2009).

\subsubsection{Slip Device Test}

During walking, the slip device test has been used to measure reactive balance in individuals with iSCI. This method, used by Arora et al. (2020), tested reactive balance in response to a slip perturbation during normal walking. During the test, participants were secured by a safety harness, which allowed for free movement during normal walking. In the middle of a 10m walkway, a set of low friction steel rollers embedded to be flush with the ground made up what was known as the "slip device". After performing at least 3 normal unperturbed walking trials where the rollers locked in place, the slip device was unlocked causing an unpredictable slip perturbation in the anterior-posterior direction. 


\subsubsection{Lean-and-Release Test}

Another measure of reactive balance, the Lean-and-Release test, measures reactive stepping by simulating a forward fall from a standing position (Chan et al., 2020; Do et al., 1982). The Leanand-Release test has been widely used to quantify reactive stepping ability for individuals with stroke (Mansfield et al., 2013) and recently has also been used for individuals with iSCI (Chan et al., 2020). During the Lean-and-Release test participants donned a safety harness connected to a ceiling attachment that provided support in the event of a fall. A horizontal cable attached to the harness lead to a force transducer and release mechanism. Once positioned on the force plate system, the participants were instructed to lean forward while maintaining extension at the hips until $8-12 \%$ of their body weight was being supported by the cable. Previous research has demonstrated $8-12 \%$ as the weight requiring a reactive step response upon release of the cable (Mansfield et al., 2011; Mansfield et al., 2013). After confirming a reading of 8-12\% from the force transducer, a researcher manually released the cable, initiating a fall in the anterior direction. In order to minimize anticipatory responses during the test, "sham" trials (i.e., no release occurs) were included at random (Chan et al. 2020).

Although the Lean-and-Release test described above involves the use of technically complex instruments (i.e. force transducer and force plate system), previous studies have used a simplified version with minimal instrumentation (Borrelli et al., 2019). Unlike the moving platform and slip device tests that may be difficult to administer clinically, the Lean-and-Release test can be simplified and therefore has potential for use in clinical settings. 


\subsubsection{Psychometric Properties of the Lean-and-Release Test}

Understanding the psychometric properties of an outcome measure helps us to determine the quality of information it is providing (Roach, 2006). In the case of the Lean-and-Release test, the information gathered from this measurement tool may help guide the development of targeted therapies to improve reactive balance among individuals with iSCI. Therefore, it is important to evaluate the psychometric properties of the Lean-and-Release test. The reliability of an outcome measure is determined based on the degree to which it produces the same value each time it is used, assuming no real change has occurred (Streiner et al., 2015). More specifically, test-retest reliability expresses the degree a test can reproduce the same measured value from one session to the next assuming no change in the test subject's status has occurred (Mokkink et al., 2010), and agreement expresses the degree of accuracy a measurement tool has by using measurement error from one session to the next (de Vet et al., 2006). The validity of an outcome measure represents how well a certain test measures what it is meant to measure (Streiner et al., 2015). The convergent validity can be expressed by demonstrating relationships between the measurement of interest and other outcome measures known to measure similar parameters (Streiner et al., 2015).

Recently, a study by Saumur et al. (2021), examining the test-retest reliability of the temporal measures of reactive stepping during the Lean-and-Release test, was conducted on a group of healthy adults. The results of this study found good to excellent test-retest reliability for foot-off (start of reactive step) and swing time of the reactive step using the lean-and-release test (Saumur et al., 2021). Additionally, all of the temporal measures demonstrated good absolute reliability other than time to restabilization (Saumur et al., 2021). However, a measurement's degree of reliability for one particular group may not translate to another group with unique characteristics 
(Roach, 2006). Therefore, in order to understand the psychometric properties of the Lean-andRelease test as they relate to people with iSCI it must be tested for reliability and validity with those individuals specifically. In a 2020 study by Unger at al., the Lean-and-Release test was found to have good test-retest reliability for measuring behavioural responses (i.e., single-step, multi-step, fall) when used on individuals with iSCI. Additionally, the test was found to be a valid measure as evidenced by positive moderate correlations between the behavioural responses and other related measures including lower extremity strength and balance confidence (Unger et al., 2020). The reliability of the temporal measures was also examined; however, unlike $\mathrm{AB}$ individuals, the only parameter found to be reliable in people with iSCI was foot-contact time (i.e., end of reactive step) (Saumur et al., 2021; Unger et al., 2020). To date, the psychometric properties of the spatial measures (i.e., step length and step width) of reactive stepping during the Lean-and-Release test have not been examined for people with iSCI.

\subsection{Reactive Balance Training}

As a result of observed behavioural differences in reactive stepping ability between individuals with iSCI and $\mathrm{AB}$ individuals, balance training interventions for people with iSCI have recently started to focus on reactive stepping (Unger et al., 2021). Conventionally, balance training focuses on anticipatory balance strategies, which involve muscle activation in anticipation of preventing a loss of balance before a voluntary movement (Unger et al., 2019). In contrast, perturbation-based balance training (PBT) targets an individual's reactive balance impairments (Unger et al., 2019). Previous research has demonstrated PBT to be an effective intervention for older adults and individuals who have had a stroke (Mansfield et al., 2015; Mansfield et al., 2011; Mansfield et al., 2010). To evaluate the effectiveness of these interventions, stepping performance during the Lean-and-Release test was used as an outcome measure. After PBT, 
individuals with stroke improved the behavioural (i.e., less steps) and temporal (i.e., reaction time) parameters of their response (Mansfield, 2011; Mansfield 2010). For older adults, evidence from multiple preliminary randomized controlled trials suggests that PBT likely reduces the risk and frequency of falls (Mansfield et al., 2015). For individuals with iSCI, PBT also resulted in significant improvements in the behavioural and temporal characteristics of reactive stepping however, similar levels of improvement were also achieved using dose-matched conventional balance training (Unger et al., 2021). The effects of PBT on the spatial characteristics of reactive stepping have yet to be examined using the Lean-and-Release test. Therefore, it is important to develop a greater understanding of the spatial characteristics of reactive stepping in people with iSCI and determine the reliability and validity of the Lean-and-Release test for measuring these spatial parameters. 


\section{References}

Arora, T., Musselman, K. E., Lanovaz, J., Linassi, G., Arnold, C., Milosavljevic, S., Oates, A. (2020). Reactive balance responses to an unexpected slip perturbation in individuals with incomplete spinal cord injury. Clinical Biomechanics, 78, 105099.

Beloozerova, I. N., Sirota, M. G., Orlovsky, G. N., Deliagina, T. G. (2005). Activity of pyramidal tract neurons in the cat during postural corrections. Journal of Neurophysiology, 93, 1831-1844.

Bone, M. D., Arora, T., Musselman, K. E., Lanovaz, J. L., Linassi, G. A., \& Oates, A. R. (2021). Investigating proactive balance control in individuals with incomplete spinal cord injury while walking on a known slippery surface. Neuroscience letters, 749, 135744.

Borrelli, J. R., Junod, C. A., Inness, E. L., Jones, S., Mansfield, A., \& Maki, B. E. (2019). Clinical assessment of reactive balance control in acquired brain injury: A comparison of manual and cable release-from-lean assessment methods. Physiotherapy Research International, 24(4), e1787.

Brotherton, S. S., Krause, J. S., \& Nietert, P. J. (2007). Falls in individuals with incomplete spinal cord injury. Spinal Cord. 45(1), 37-40.

Chan, K., Lee, J. W., Unger, J., Yoo, J., Masani, K., \& Musselman, K. E. (2020). Reactive stepping after a forward fall in people living with incomplete spinal cord injury or disease. Spinal Cord. 58, 185-193. 
Chiba, R., Takakusaki, K., Ota, J., Yozu, A., Haga, N. (2016). Human upright posture control models based on multisensory inputs; in fast and slow dynamics. Neuroscience Research, 2014, 96-104.

Day, K. V., Kautz, S. A., Wu, S. S., Suter, S. P., Behrman, A. L. (2011). Foot placement variability as a walking balance mechanism post-spinal cord injury. Clinical Biomechanics, $13,13$.

de Vet, H. C., Terwee, C. B., Knol, D. L., \& Bouter, L. M. (2006). When to use agreement versus reliability measures. Journal of Clinical Epidemiology, 59(10), 1033-1039.

Do, M. C., Breniere, Y., \& Brenguier, P. (1982). A biomechanical study of balance recovery during the fall forward. Journal of Biomechanics, 15(12), 933-939.

Doucet, B. M., Lam, A., \& Griffin, L. (2012). Neuromuscular electrical stimulation for skeletal muscle function. The Yale Journal of Biology and Medicine, 85(2), 201-215.

Hageman, P. A., \& Blanke, D. J. (1986). Comparison of gait of young women and elderly women. Physical Therapy, 66(9), 1382-1387.

Hamid, S., \& Hayek, R. (2008). Role of electrical stimulation for rehabilitation and regeneration after spinal cord injury: An overview. European Spine Journal, 17(9), 1256-1269.

Hilliard, M. J., Martinez, K. M., Janssen, I., Edwards, B., Mille, M. L., Zhang, Y., \& Rogers, M. W. (2008). Lateral balance factors predict future falls in community-living older adults. Archives of Physical Medicine and Rehabilitation, 89(9), 1708-1713.

Hof, A. L., Gazendam, M. G., \& Sinke, W. E. (2005). The condition for dynamic stability. Journal of biomechanics, 38(1), 1-8. 
Horak, F. B. (2006). Postural orientation and equilibrium: what do we need to know about neural control of balance to prevent falls? Age and Ageing, 35(2), ii7-ii11.

Houston, D., Unger, J., Lee, J. W., Masani, K., Musselman, K. E. (2020). Functional electrical stimulation plus visual feedback balance training for standing balance performance among individuals with incomplete spinal cord injury: a case series. Frontiers in Neurology Neurorehabilitation, 11, 680.

Hsiao-Wecksler, E. T., \& Robinovitch, S. N. (2007). The effect of step length on young and elderly women's ability to recover balance. Clinical Biomechanics (Bristol, Avon), 22(5), $574-580$.

Jørgensen, V., \& Roaldsen, K. S. (2017). Negotiating identity and self-image: perceptions of falls in ambulatory individuals with spinal cord injury - a qualitative study. Clinical Rehabilitation, 31(4), 544-554.

Jørgensen, V., Butler Forslund, E., Opheim, A., Franzén, E., Wahman, K., Hultling, C., Seiger, Å., Ståhle, A., Stanghelle, J. K., \& Roaldsen, K. S. (2017). Falls and fear of falling predict future falls and related injuries in ambulatory individuals with spinal cord injury: a longitudinal observational study. Journal of Physiotherapy, 63(2), 108-113.

Khan, A., Pujol, C., Laylor, M., Unic, N., Pakosh, M., Dawe, J., \& Musselman, K. E. (2019). Falls after spinal cord injury: A systematic review and meta-analysis of incidence proportion and contributing factors. Spinal Cord. 57(7), 526-539. 
Krueger, H., Noonan, V., Trenaman, L., Joshi, P., \& Rivers, C. (2013). The economic burden of traumatic spinal cord injury in Canada. Chronic Diseases and Injuries in Canada, 33(3), $113-122$.

Kumar, R., Lim, J., Mekary, R. A., Rattani, A., Dewan, M. C., Sharif, S. Y., Osorio-Fonseca, E., \& Park, K. B. (2018). Traumatic spinal injury: Global epidemiology and worldwide volume. World Neurosurgery, 113, e345-e363.

Lemay, J. F., Gagnon, D. H., Nadeau, S., Grangeon, M., Gauthier, C., Duclos, C. (2014). Centerof-pressure total trajectory length is a complementary measure to maximum excursion to better differentiate multidirectional standing limits of stability between individuals with incomplete spinal cord injury and able-bodied individuals. Journal of NeuroEngineering and Rehabilitation, 11, 8.

Lemay, J. F., Gagnon, D., Duclos, C., Grangeon, M., Gauthier, C., Nadeau, S. (2013). Influence of visual inputs on quasi-static standing postural steadiness in individuals with spinal cord injury. Gait \& Posture, 38, 357-360.

Lemay, J. F., Noamani, A., Unger, J., Houston, D. J., Rouhani, H., Musselman, K. E. (2020). Using wearable sensors to characterize gait after spinal cord injury: evaluation of testretest reliability and construct validity. Spinal Cord, E-pub Oct 6.

Maki, B. E., \& McIlroy, W. E. (1997). The role of limb movements in maintaining upright stance: the "change-in-support" strategy. Physical therapy, 77(5), 488-507.

Maki, B. E., \& Mcilroy, W. E. (2006). Control of rapid limb movements for balance recovery: Age-related changes and implications for fall prevention. Age Ageing, 35(2), ii12-ii18. 
Mansfield, A., Inness, E. L., Komar, J., Biasin, L., Brunton, K., Lakhani, B., \& McIlroy, W. E. (2011). Training rapid stepping responses in an individual with stroke. Physical Therapy, 91(6), 958-969.

Mansfield, A., Inness, E. L., Lakhani, B., \& McIlroy, W. E. (2012). Determinants of limb preference for initiating compensatory stepping post- stroke. Archives of Physical Medicine and Rehabilitation, 93(7), 1179-1184.

Mansfield, A., Inness, E. L., Wong, J. S., Fraser, J. E., \& McIlroy, W. E. (2013). Is impaired control of reactive stepping related to falls during inpatient stroke rehabilitation? Neurorehabilitation and Neural Repair, 27(6), 526-533.

Mansfield, A., Peters, A. L., Liu, B. A., \& Maki, B. E. (2010). Effect of a perturbation-based balance training program on compensatory stepping and grasping reactions in older adults: A randomized controlled trial. Physical Therapy, 90(4), 476-491.

Mansfield, A., Wong, J. S., Bryce, J., Knorr, S., \& Patterson, K. K. (2015). Does perturbationbased balance training prevent falls? Systematic review and meta-analysis of preliminary randomized controlled trials. Physical therapy, 95(5), 700-709.

McCrum, C., Gerards, M. H. G., Karamanidis, K., Zijlstra, W., \& Meijer, K. (2017). A systematic review of gait perturbation paradigms for improving reactive stepping responses and falls risk among healthy older adults. European Review of Aging and Physical Activity, 14(1). 
Medell, J. L., \& Alexander, N. B. (2000). A clinical measure of maximal and rapid stepping in older women. The Journals of Gerontology Series A: Biological Sciences and Medical Sciences, 55(8), M429-M433.

Mokkink, L. B., Terwee, C. B., Patrick, D. L., Alonso, J., Stratford, P. W., Knol, D. L., Bouter, L. M., \& de Vet, H. C. (2010). The COSMIN study reached international consensus on taxonomy, terminology, and definitions of measurement properties for health-related patient-reported outcomes. Journal of Clinical Epidemiology, 63(7), 737-745.

Musselman, K. E., Arnold, C., Pujol, C., Lynd, K., \& Oosman, S. (2018). Falls, mobility and physical activity after spinal cord injury: An exploratory study using photo-elicitation interviewing. Spinal Cord Series and Cases. 4, 39.

Musselman, K. E., Unger, J., \& Lemay, J. F. (2021). Assessment of postural control after spinal cord injury or disease: A narrative review. In R. Rajendram, V. R. Preedy, \& C. R. Martin (Eds.), Diagnosis and treatment of spinal cord injury. 1st Edition. Elsevier Inc. In press.

Musselman, K.E., Singh, H., \& Unger, J. (2019). Shaping rehabilitation after spinal cord injury: The impact of qualitative research. In C. M. Hayre \& D. J. Müller (Eds.), Enhancing healthcare and rehabilitation: The impact of qualitative research. Crc Press, Taylor \& Francis Group.

New, P. W., Farry, A., Baxter, D., \& Noonan, V. K. (2013). Prevalence of non-traumatic spinal cord injury in Victoria, Australia. Spinal cord, 51(2), 99-102. 
New, P. W., Guilcher, S. J. T., Jaglal, S. B., Biering-Sørensen, F., Noonan, V. K., \& Ho, C. (2017). Trends, challenges, and opportunities regarding research in non-traumatic spinal cord dysfunction. Topics in Spinal Cord Injury Rehabilitation, 23(4), 313-323.

Noamani, A., Lemay, J. F., Musselman, K. E., Rouhani, H. (2020). Postural control strategy after incomplete spinal cord injury: effect of sensory inputs on trunk-leg movement coordination. Journal of NeuroEngineering and Rehabilitation, 17, 141.

Oberg, T., Karsznia, A., \& Oberg, K. (1993). Basic gait parameters: reference data for normal subjects, 10-79 years of age. Journal of Rehabilitation Research and Development, 30(2), $210-223$.

Pollock, A. S., Durward, B. R., Rowe, P. J., \& Paul, J. P. (2000). What is balance? Clinical Rehabilitation, 14, 402-406.

Roach, K. E. (2006). Measurement of Health Outcomes: Reliability, Validity and Responsiveness. JPO Journal of Prosthetics and Orthotics, 18(Proceedings), P8-P12.

Saumur, T. M., Mathur, S., Nestico, J., Perry, S. D., Mochizuki, G., \& Mansfield, A. (2021). Test-retest reliability of force plate-derived measures of reactive stepping. Journal of Biomechanics, 115, 110185.

Sibley, K. M., Beauchamp, M. K., Van Ooteghem, K., Straus, S. E., \& Jaglal, S. B. (2015). Using the systems framework for postural control to analyze the components of balance evaluated in standardized balance measures: a scoping review. Archives of Physical Medicine and Rehabilitation, 96(1), 122-132. 
Singh, H., Chan, K., Cheung, L., Hitzig, S., Musselman, K.E. (2021*). The impact of falls and fear of falling on participation, autonomy, and life satisfaction among individuals with spinal cord injury: a brief report. Journal of Spinal Cord Medicine, Under Review.

Singh, H., Shibi Rosen, A., Bostick, G., Kaiser, A., \& Musselman, K. E. (2020). Exploring the causes and impacts of falls among ambulators with spinal cord injury using photovoice: A mixed methods study. BMJ Open. 10, e039763.

Streiner, D. L., Norman, G. R., \& Cairney, J. (2015). Health measurement scales: A practical guide to their development and use. Oxford University Press.

Thigpen, M. T., Cauraugh, J., Creel, G., Day, K., Flynn, S., Fritz, S., Frost, S., Respess, R., Gardner-Smith, P., Brack, M., Behrman, A. (2009). Adaptation of postural responses during different standing perturbation conditions in individuals with incomplete spinal cord injury. Gait \& Posture, 29, 113-8.

Unger, J., Chan, K., Lee, J. W., Craven, B. C., Mansfield, A., Alavinia, M., Masani, K., \& Musselman, K. E. (2021). The effect of Perturbation-based Balance Training and Conventional Intensive Balance Training on reactive stepping ability in individuals with incomplete spinal cord injury or disease: A randomized clinical trial. Frontiers in Neurology, 12, 620367.

Unger, J., Chan, K., Scovil, C. Y., Craven, B. C., Mansfield, A., Masani, K., \& Musselman, K. E. (2019). Intensive balance training for adults with incomplete spinal cord injuries: Protocol for an assessor-blinded randomized clinical trial. Physical Therapy, 99(4), 420427. 
Unger, J., Oates, A. R., Lanovaz, J., Chan, K., Lee, J. W., Theventhiran, P., Masani, K., \& Musselman, K. E. (2020). The measurement properties of the Lean-and-Release test in people with incomplete spinal cord injury or disease. The Journal of Spinal Cord Medicine, 1-10.

Wierbecky, J., \& Kamath, A. (2013). Pathophysiology of spinal cord injury. In S. Nesathurai (Ed.), The rehabilitation of people with spinal cord injury (pp. 15-17). Pubblicazione.

Zelenin, P. V., Beloozerova, I. N., Sirota, M. G., Orlovsky, G. N., Deliagina, T. G. (2010). Activity of red nucleus neurons in the cat during postural corrections. Journal of Neuroscience, 30, 14533-14542. 


\title{
Chapter 3
}

\section{Spatial Characteristics of Reactive Stepping Among People Living with Chronic Incomplete Spinal Cord Injury}

\author{
Heffernan, M. G., Lee, J. W., Chan, K., Unger, J., Marzolini, S., Welsh, T. N., Masani, K., \\ Musselman, K. E. (2021) Spatial characteristics of reactive stepping among people living with \\ chronic incomplete spinal cord injury. Spinal Cord, Manuscript submitted for review.
}

\subsection{Introduction}

People with incomplete spinal cord injuries (iSCI) have impaired balance and, as a result, falling is a serious issue for these individuals (Khan et al., 2019). Previous research has demonstrated that $78 \%$ of ambulatory people with iSCI experience one or more falls each year and approximately 50\% experience repeated falls (Jørgensen et al., 2017; Khan et al., 2019). These falls, which often occur during walking, can have serious consequences that require immediate and costly medical attention (Brotherton et al., 2007). Additionally, secondary consequences can arise from a fall or fall related injury. For example, people who fall often develop a fear of falling that can have a significant impact on an individual's daily mobility, level of depression, autonomy, and quality of life (Jørgensen \& Roaldsen, 2017; Musselman et al., 2018; Singh et al., $2021 *$, Singh et al. 2020).

Prevention of falls requires individuals with iSCI to produce effective reactive balance responses to unexpected perturbations (Maki \& Mcilroy, 2006). Specifically, to maintain balance after an unexpected perturbation, a quick reactive step(s) can be initiated to adjust the position of an individual's centre of mass $(\mathrm{CoM})$ such that it remains within their base of support (BoS) 
(Hilliard et al., 2008; Mansfield et al., 2012; Mansfield et al., 2013; McCrum et al., 2017). Often, individuals will take an additional step when the first step does not provide sufficient compensation for the initial change in CoM (Hilliard et al., 2008; Mansfield et al., 2012; Mansfield et al., 2013; McCrum et al., 2017).

Studies that measure reactive stepping use the Lean-and-Release test: an experimental tool that simulates a forward fall from a standing position (Do et al., 1982). Originally, the Lean-andRelease test was developed to quantify reactive stepping ability for individuals following a stroke (Mansfield et al., 2013). Recently however, this tool has been used to test the reactive stepping ability of individuals with iSCI (Chan et al., 2020). The Lean-and-Release test has adequate testretest reliability when used to measure the behavioural response (i.e., single-step, multi-steps or fall) of individuals with iSCI (Unger et al., 2020). Additionally, the test is considered to be safe, and it is a standardized way to provide quantitative data of an individual's reactive step(s) (Unger et al., 2020).

A recent study demonstrated that, compared with able-bodied (AB) individuals, people with iSCI are more likely to require multiple reactive steps to regain balance after a simulated forward fall (Chan et al., 2020). However, the mechanisms contributing to these behavioural differences in reactive stepping remain unclear. Although all six leg muscles examined had a more variable onset of muscle activation for people with iSCI, only the tibialis anterior muscle of the stepping leg was consistently delayed in the onset of muscle activation compared to AB individuals (Chan et al., 2020). Additionally, no significant differences were found in the temporal parameters (i.e., movement timing) of the reactive step between individuals with iSCI and AB individuals (Chan et al., 2020). 
As a result of its importance for fall prevention, reactive stepping ability has recently become a focus of balance training interventions for people with iSCI (Unger et al., 2021). Therefore, it is important to investigate the underlying mechanisms driving behavioural differences in reactive stepping in order to develop the most effective interventions. Although previous research has compared the onset of muscle activation and temporal parameters during reactive stepping in individuals with iSCI (Chan et al., 2020), the spatial characteristics of the reactive step (i.e., step length and width) have not yet been examined. It has been suggested that the behavioural differences may be explained by a deficiency in the spatial characteristics (i.e., shorter reactive step length) (Chan et al., 2020). This suggestion has been supported by studies which have investigated the reactive stepping of older AB adults. These studies have demonstrated that, compared with younger adults, older adults take shorter reactive step lengths (Hageman \& Blanke, 1986; Hsiao-Wecksler \& Robinovitch, 2007; Medell \& Alexander, 2000; Oberg et al., 1993).

To investigate the relationship between the spatial characteristics and behavioural response of reactive stepping, we compared the spatial characteristics of reactive stepping (i.e., mean values and variability of reactive step length and width), elicited with the Lean-and-Release test, between individuals with chronic motor iSCI and age- and sex-matched AB individuals. Based on the findings of previous studies involving older adults, we hypothesized that after a simulated forward fall, individuals with iSCI would take reactive steps that are shorter in length compared to $\mathrm{AB}$ individuals. 


\subsection{Methods}

This study is a secondary analysis of cross-sectional data collected at the Lyndhurst Centre, Toronto Rehabilitation Institute, University Health Network. This study was part of a larger research study evaluating the effects of perturbation-based balance training on balance ability in participants with iSCI (ClinicalTrials.gov identifier NCT02960178) (Unger at al., 2021). Ethical approval for this study was granted by the University Health Network Research Ethics Board and written informed consent was obtained from each participant.

\subsubsection{Participants}

A central recruitment database containing the names of individuals with iSCI who were inpatients at the Lyndhurst Centre was used to recruit participants for this study. These individuals had previously consented to receive notifications regarding ongoing research studies. Recruitment flyers for the study were also posted at the Lyndhurst Centre.

Individuals who were at least 18 years old and diagnosed with a chronic (i.e., >1-year postinjury), traumatic or non-traumatic and non-progressive iSCI were eligible to participate. They were required to have a level C or D injury as defined by the American Spinal Injury Association Impairment Scale (AIS), a moderate level of trunk control (i.e., able to reach forward with their arms a minimum of 2 inches in an unsupported standing position) (Berg et al., 1995) and be able to stand independently for 30 seconds. They were also required to be free of any other conditions that affected their balance and/or walking ability. Individuals were excluded from participation in the study if they could not achieve a standing position due to contractures or spasticity, had a pressure injury of grade 2 or greater on the pelvis or trunk, and/or had a history of fragility fracture(s) in the lower extremities. 
Sex- and age-matched ( \pm 3 years) AB participants were recruited through emails sent to listservs of the Lyndhurst Centre. AB individuals were excluded from participation if they had a condition or injury that affected balance/walking, had a history of fragility fracture(s) in the lower extremities, or used a gait aid or ankle-foot orthosis to ambulate independently.

\subsubsection{Experimental Procedure}

The setup and configuration of the Lean-and-Release test is illustrated in Figure 3.1. During the Lean-and-Release trials participants wore a safety harness connected to a ceiling attachment which provided support in the event of a loss of balance. Attached to the safety harness at waist height was a horizontal cable which led to a force transducer (MLP-100-CO-C, Transducer Techniques, Temecula, USA) and a custom-made release mechanism. The participants were positioned on a dual force plate system consisting of four, $25.1 \times 50.2 \mathrm{~cm}$ components (AccuSway Dual, Advanced Mechanical Technology Inc., Watertown, USA) such that each foot was on one of the two components closest to the cable and release mechanism. Once positioned, the participants were instructed to lean forward while maintaining extension at the hips until the researcher monitoring the output of the force transducer confirmed that $8-12 \%$ of their body weight was being supported by the cable. The $8-12 \%$ target was determined, based on previous research, to be the weight that requires a reactive step response in order to maintain balance after release of the cable (Mansfield et al., 2015; Unger et al., 2020). Then a researcher positioned behind the participant manually released the cable sometime within the following 30 seconds, initiating a fall in the anterior direction During a testing session, participants completed up to ten Lean-and-Release trials with three "sham" trials included randomly (i.e. participants held a forward lean position, but the cable was not released) to reduce anticipatory responses. 


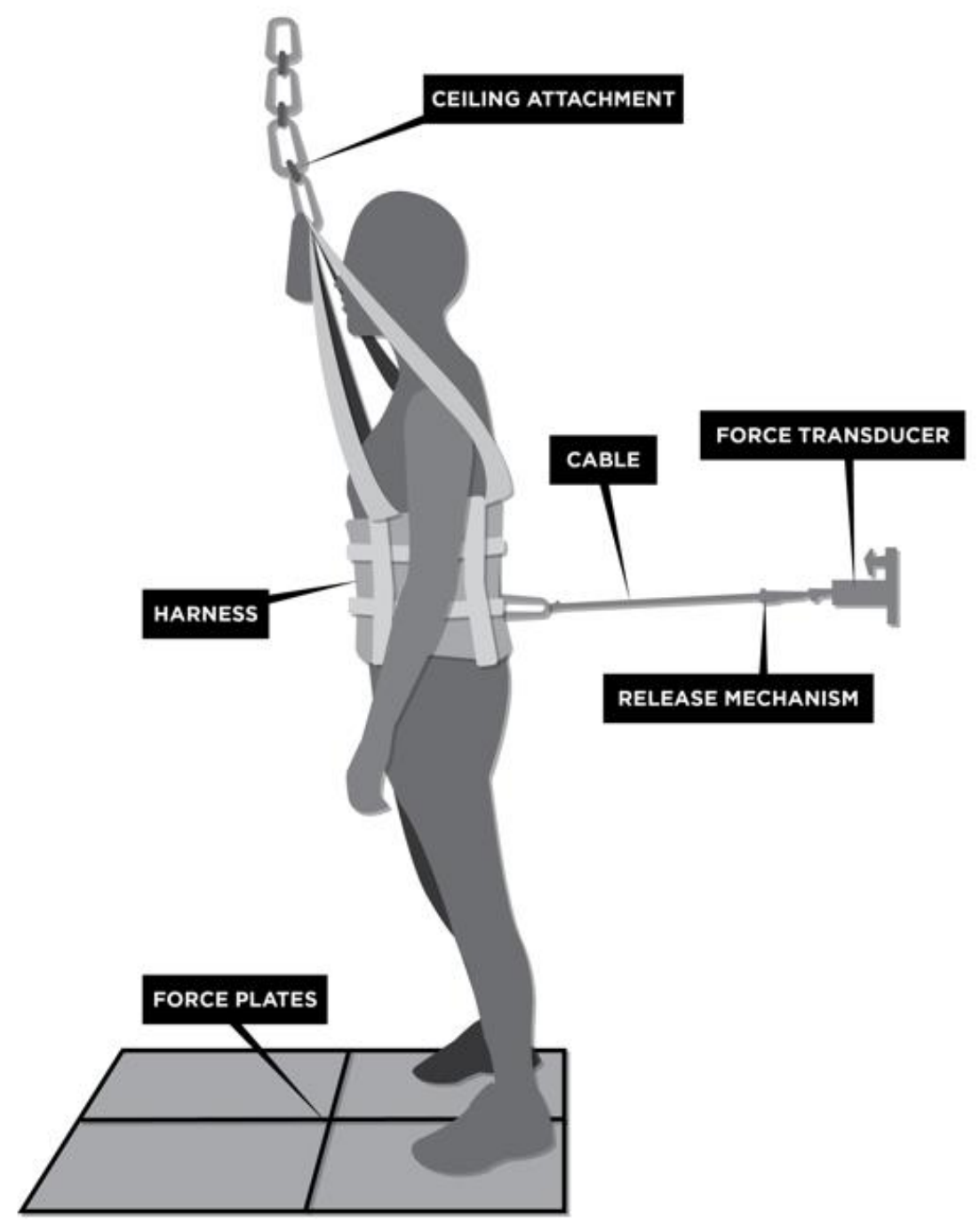

Figure 3.1. Configuration of the Lean-and-Release test (Chan et al., 2020). Copyright permission granted.

\subsubsection{Data Acquisition}

Each participant's age and sex were collected upon enrollment in the study. Additionally, participants in the iSCI group provided their AIS score, neurological level of injury, time postinjury, cause of injury, and use of gait aid for daily ambulation via self-report. iSCI participants were also asked to provide a retrospective account of their fall history as defined by any incident 
in the last three months where they came to rest on the ground or another lower surface unintentionally (World Health Organization, 2018).

Kinetic data were collected using the force plate system and recorded using the data acquisition system integrated in the motion capture system described below (Cortex ver. 3.1, Motion Analysis Corp., USA) at a sampling frequency of $2000 \mathrm{~Hz}$. Force plate data were used to determine participant mass as well as the temporal parameters of the reactive step. Specifically, the foot-off (i.e., start of reactive step) and foot contact times (i.e., end of reactive step) of the stepping leg were identified.

Kinematic data were collected using a 3D motion capture system that included six cameras (Rapter-E; Motion Analysis Corp., USA) and 35 reflective markers attached at anatomical landmarks including two that were secured to the participant's shoes at each heel. Kinematic data were recorded at a sampling frequency of $200 \mathrm{~Hz}$ and were used to determine participant height as well as the spatial parameters of the first reactive step. Specifically, the length and width of the first reactive step were calculated.

Behavioural data were collected by a researcher who observed the participant's response for each trial of the Lean-and-Release test. Responses were classified as either single-step (i.e., a single foot contact after foot-off), multi-step (i.e., multiple foot contacts required to recover balance after foot-off), or a fall (balance was not recovered or assistance from therapist was required) (Unger et al., 2020). 


\subsubsection{Data Analysis}

Mean ( \pm 1 standard deviation) age and time post-injury were reported. Sex, AIS score, neurological level of injury, cause of injury, and use of gait aid and/or braces were reported as a frequency count.

All kinematic and kinetic data were processed using a custom-written script in a computational software (MATLAB 2018a; MathWorks, USA). The force plate data were filtered using a 4th order low-pass Butterworth filter with a cut-off frequency of $30 \mathrm{~Hz}$. Foot-off time was determined by the occurrence of $<1 \%$ of the participants body weight registering on the force plate under the stepping foot after the cable was released. Foot contact time was determined by the occurrence of $>1 \%$ of the participant's body weight registering on any force plate under the stepping foot after foot-off time. As illustrated in Figure 3.2, step length was calculated by measuring the change in anterior-posterior positions of the heel marker of the stepping foot from foot-off time to the time of foot contact. Step width was calculated by measuring the change in medial-lateral positions of the heel marker of the stepping foot from foot-off time to the time of foot contact. A negative step width value represents a step with narrower BoS. Similar studies, investigating reactive stepping in older adults and individuals with stroke, have calculated the spatial characteristics of the reactive steps using analogous measurements (Martinez et al., 2019; Weaver, 2017). Step length and width were measured in centimeters and normalized for participant height (e.g., step length $\mathrm{cm} /$ participant height $\mathrm{cm}=$ normalized step length). Finally, behavioural data were reported as a proportion of the trials where single-step, multi-step and fall responses occurred. 
Length

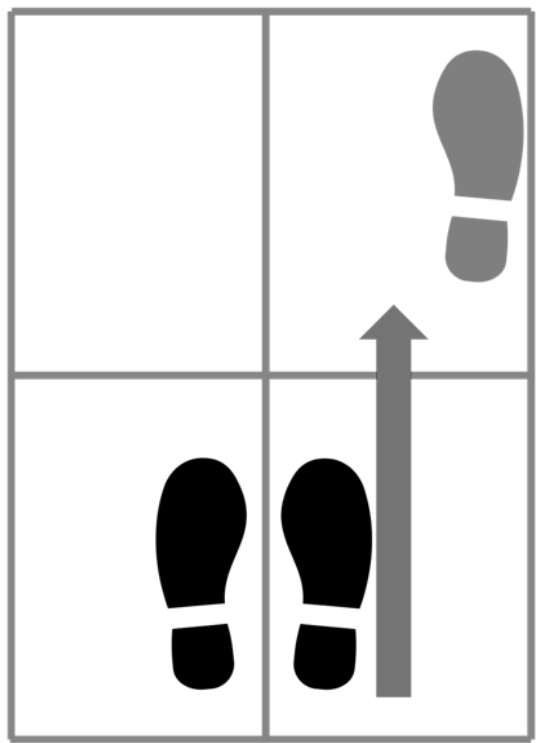

Width

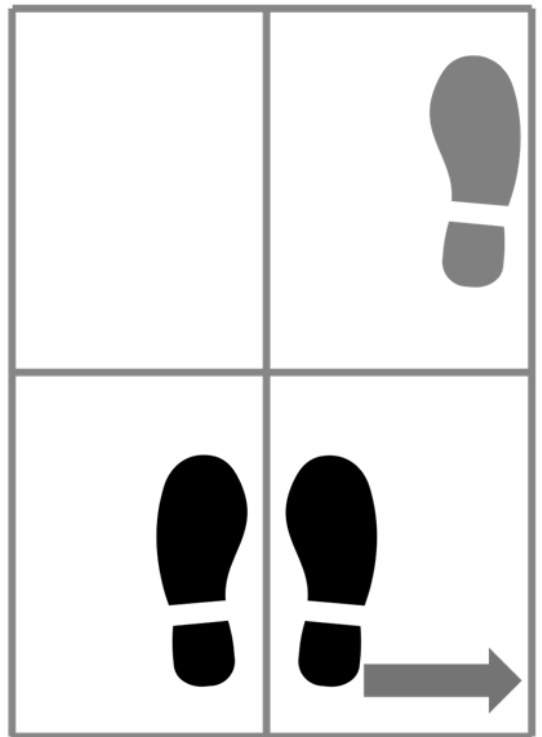

Figure 3.2 Spatial characteristics of reactive stepping measured using starting heel marker position at foot-off time (black footprint) and heel marker position after first step at foot contact time (grey footprint). Arrows represent direction of measurement. Rectangles represent force plate configuration.

The mean ( \pm 1 standard deviation) for normalized step length and width were calculated for the iSCI and AB groups. Additionally, the variability of these spatial parameters was represented by the mean standard deviation for each group. To calculate the mean standard deviation for each spatial parameter, the standard deviation within a participant (i.e., across trials) was first calculated. Next, the standard deviations from the participants in the same group (i.e., iSCI or AB) were combined and averaged. Variability is often represented by coefficient of variation. However, our measurements produced negative values (i.e. steps that narrowed BoS) that prevented a valid calculation of coefficient of variation.

Data sets were tested for normality using a Shapiro-Wilk test and for equality of variances using a Levene's test. Since assumptions concerning normality and equality of variances were met, the spatial parameters were compared between the iSCI and AB groups using independent t-tests. The effect size was calculated using Cohen's d. Effect sizes were interpreted as either small $(\mathrm{d}=$ 
$0.2)$, medium $(\mathrm{d}=0.5)$ or large $(\mathrm{d}=0.8)$. As a result of the observed differences in variability of the spatial parameters between groups, additional analyses were conducted. Specifically, among the participants with iSCI, Spearman and point-biserial correlation coefficients were used to explore the relationship between the variability of the spatial parameters and 1) the behavioural responses on the Lean-and-Release test (i.e., proportion of trials with a single-step response and proportion of trials with a fall response), and 2) categorical 3-month fall history (i.e., $1=$ one or more falls reported, $0=$ no falls reported). Data were analyzed using SPSS 25 (IBM, Armonk, NY, USA). Alpha was set to 0.05 .

\subsection{Results}

\subsubsection{Participants}

Twenty-one individuals with incomplete iSCI participated in the study, however a total of 12 participants were included in the current analysis. Participant loss occurred as a result of inability to execute any reactive steps during Lean-and-Release test $(n=4)$, missing or faulty force plate or motion capture data $(n=4)$. Additionally, one participant ( 87 years old, male) was not included in our analyses because an age- and sex-matched $\mathrm{AB}$ participant could not be recruited. The iSCI group included nine females and three males with a mean age of 53.6 (15.2) years. Most of the participants with iSCI (75\%) were able to complete 10 trials of the Lean-and-Release test. One participant completed nine trials, one completed eight and one completed six (not including additional 'sham' trials). A total of 11 age- and sex-matched AB participants were included. One AB participant was age- and sex-matched to two iSCI participants. The AB group included eight females and three males with a mean age of 54.8 (14.0) years. All demographic, injury, and fall related data are reported in Table 3.1 . 
Table 3.1. Demographic, injury and fall history data for iSCI participants.

\begin{tabular}{ccccccc}
\hline ID \# & Sex & $\begin{array}{c}\text { Age } \\
\text { (years) }\end{array}$ & $\begin{array}{c}\text { Time post- } \\
\text { injury (years) }\end{array}$ & $\begin{array}{c}\text { Level of } \\
\text { injury }\end{array}$ & $\begin{array}{c}\text { Walking } \\
\text { aid }\end{array}$ & $\begin{array}{c}\text { Fall } \\
\text { history }\end{array}$ \\
\hline PBT 5 & F & 32 & 3.5 & C4 & None & 1 \\
PBT 6 & M & 70 & 1.8 & T1 & Cane & 0 \\
PBT 8 & M & 60 & 3.2 & C5 & None & 0 \\
PBT 10 & F & 43 & 3.9 & T6 & None & 1 \\
PBT 12 & F & 87 & 2.6 & T4 & Cane & 0 \\
PBT 13 & F & 57 & 2.9 & C2 & Cane & 0 \\
PBT 14 & F & 59 & 1.1 & C1 & 4WW & 0 \\
PBT 16 & F & 55 & 9.1 & C5 & Cane & 1 \\
PBT 17 & F & 38 & 1.3 & T4 & 4 WW & 1 \\
PBT 23 & F & 38 & 6.8 & T11 & None & 1 \\
PBT 24 & M & 51 & 7.9 & C3 & Poles/4WW & 0 \\
PBT 25 & F & 53 & 39 & C4 & Poles & 1 \\
\hline Mean & - & 53.6 & 4.0 & - & - & - \\
(SD) & & $(15.2)$ & $(2.7)$ & & & 6 \\
\hline Count & M & - & - & - & - & \\
\hline Fall His & & & & & \\
\hline
\end{tabular}

Fall History: $1=$ fall in last 3-months, $0=$ no falls in last 3-months

4WW: 4-wheeled walker

\subsubsection{Spatial Characteristics}

Normalized step length $(\mathrm{t}(21)=0.07, \mathrm{p}=0.94, \mathrm{~d}=0.03)$ and step width $(\mathrm{t}(21)=0.65, \mathrm{p}=0.52, \mathrm{~d}$ $=0.30$ ) were similar for both groups with small effect sizes (see Figure 3). Variability of the spatial parameters, however, differed between groups (see Figure 4). Individuals in the iSCI group had significantly greater mean standard deviations for normalized step length $(\mathrm{t}(21)=$ 2.57, $\mathrm{p}=0.02, \mathrm{~d}=1.09)$ and step width $(\mathrm{t}(21)=3.12, \mathrm{p}=0.01, \mathrm{~d}=1.36)$ and effect sizes were large. 

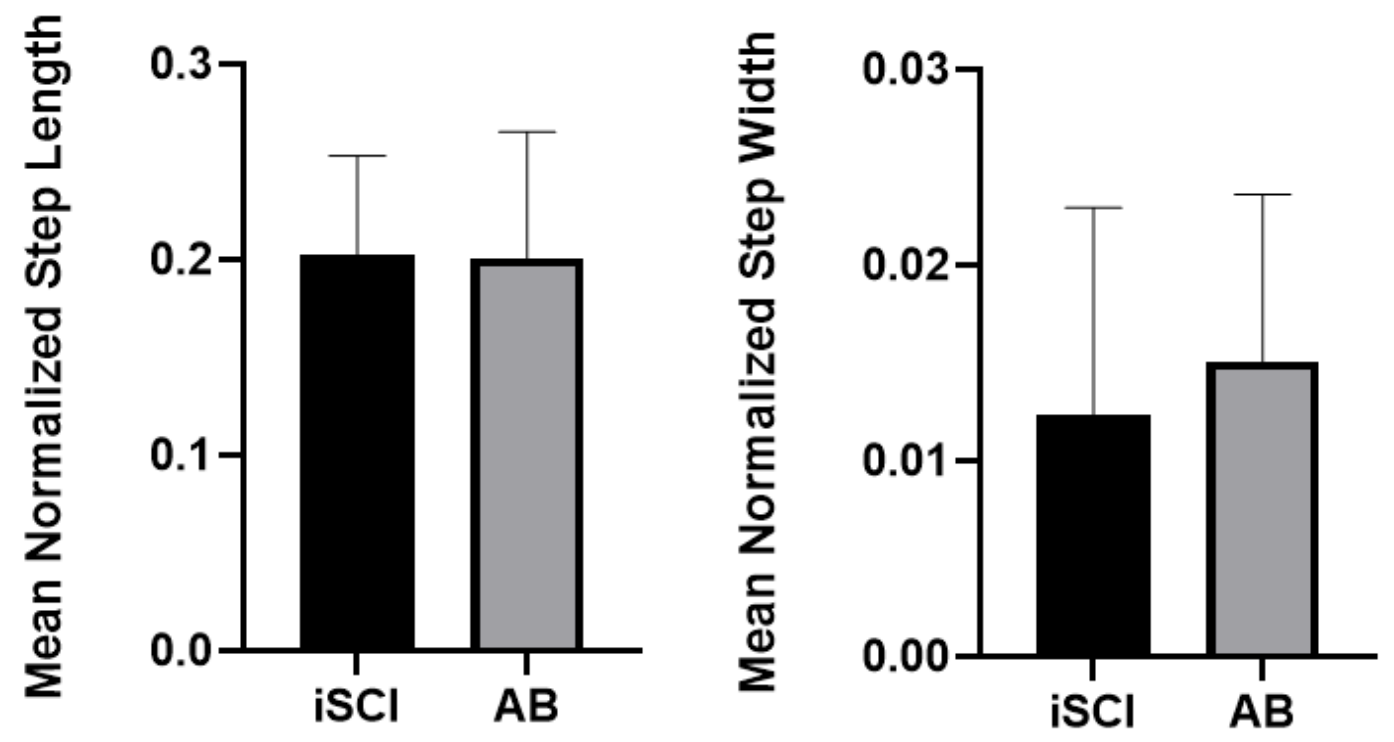

Figure 3.3. Mean step length and width normalized to participant height.
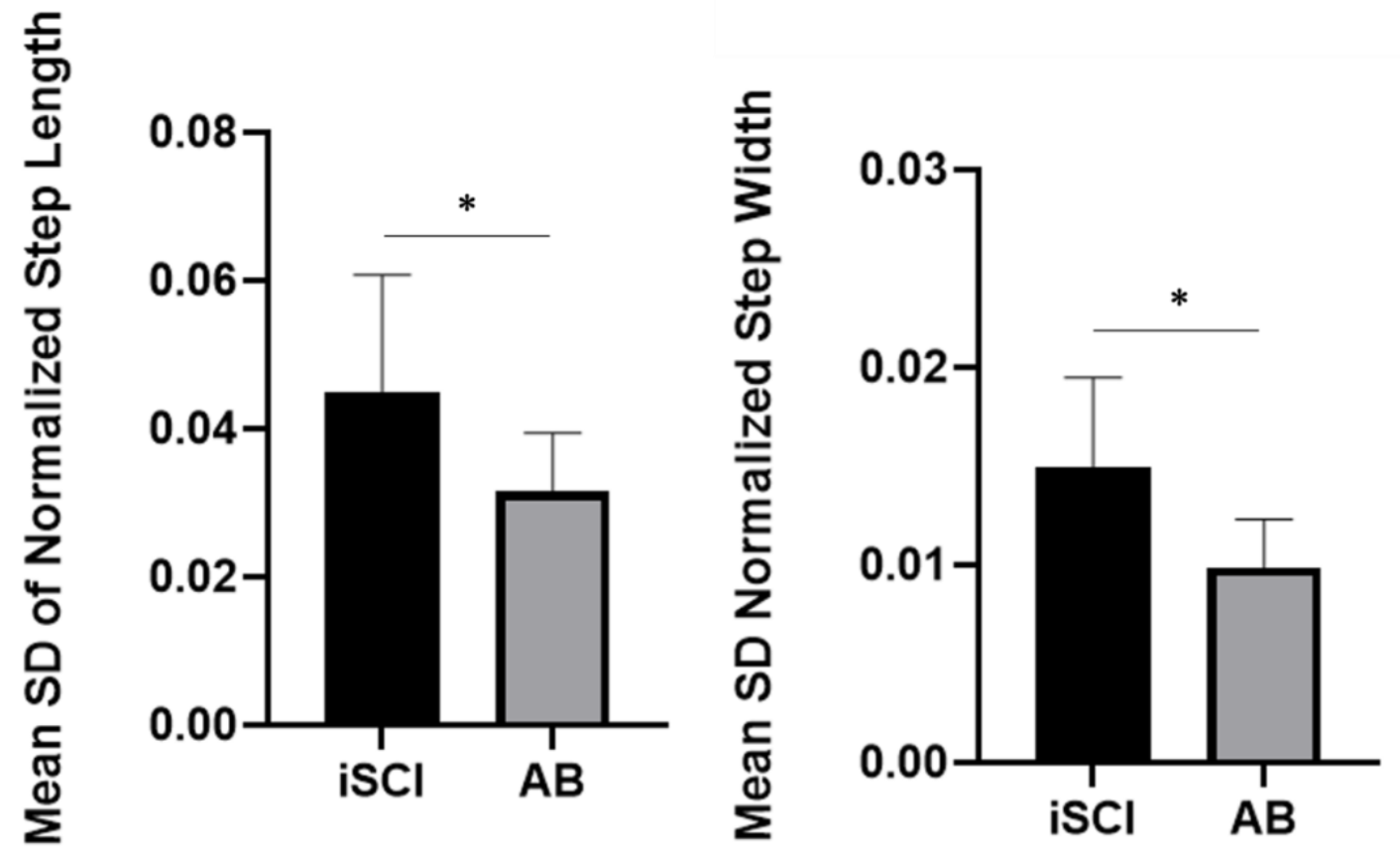

Figure 3.4. Mean standard deviation of step length and width normalized to participant height. Significant differences indicated by horizontal lines and asterisks. 


\subsubsection{Behavioural Response}

No correlation was found between the proportion of trials with a single-step response and the variability of step length $\left(\mathrm{r}_{\mathrm{s}}(10)=-0.34, \mathrm{p}=0.28\right)$ or step width $\left(\mathrm{r}_{\mathrm{s}}(10)=0.28, \mathrm{p}=0.41\right)$ among the participants with iSCI. There was also no correlation found between the proportion of trials with a fall response and the variability of step length $\left(\mathrm{r}_{\mathrm{s}}(10)=0.08, \mathrm{p}=0.81\right)$ or step width $\left(\mathrm{r}_{\mathrm{s}}(10)=0.51, \mathrm{p}=0.12\right)$ among the participants with iSCI.

\subsubsection{Fall History}

Six $(50 \%)$ of the participants in the iSCI group reported one or more falls in the previous three months. No correlation was found between 3-month fall history and the variability of step length $\left(\mathrm{r}_{\mathrm{pb}}(10)=-0.16, \mathrm{p}=0.62\right)$ or step width $\left(\mathrm{r}_{\mathrm{pb}}(10)=0.03, \mathrm{p}=0.92\right)$ among the participants with iSCI.

\subsection{Discussion}

In this study we investigated the spatial characteristics of the reactive stepping response in individuals with chronic motor iSCI. Contrary to our hypothesis, the reactive steps of individuals with iSCI were similar in length and width to the reactive steps taken by age- and sex-matched AB individuals. However, significant between-group differences in the variability of step length and step width were observed. The findings suggest individuals with iSCI are able to take a first reactive step that is similar in magnitude to their $A B$ counterparts but, from one standardized perturbation to the next, the length and width of their reactive steps have more variation.

The increased variability in the spatial characteristics of reactive stepping may indicate that individuals with iSCI take some reactive steps that are too long or wide, and others that are not 
long or wide enough. In doing so they fail to consistently achieve an ideal repositioning of their CoM within their base of support, leading to falls and multi-step reactive responses. One potential method for quantifying the sufficiency of the length and width of a reactive step is to calculate margin of stability. Margin of stability, the distance between an individual's extrapolated CoM and the borders of their BoS, is a useful measure during dynamic situations such as the Lean-and-Release test (Hof et al., 2005). Individuals can increase their BoS after a forward fall through reactive stepping responses (Hilliard et al., 2008; Mansfield et al., 2012; Mansfield et al., 2013; McCrum et al., 2017), thereby increasing their margin of stability. In prior work, individuals with iSCI demonstrated an impaired ability to increase their margin of stability in the lateral direction (i.e., step width) when responding to a slip perturbation during overground walking (Arora et al., 2020). Future research involving the Lean-and-Release test could include measurement of margin of stability in the anterior and lateral directions to further investigate the implications of increased variability of reactive step lengths and widths.

Increased variability in other parameters of reactive stepping have been reported. Chan et al. (2020), found that the onset of lower limb muscle activation during a reactive step was more variable in participants with iSCI when compared to age- and sex-match AB individuals. Similarly, individuals with iSCI have also been shown to have greater stepping variability during a variety of different walking conditions. For example, during walking on a split-belt treadmill, people with iSCI demonstrated an increase in variability of the spatial parameters of stepping including length, width, foot placement (relative to CoM) and margin of stability (Day et al., 2011). Additionally, the walking cadence of individuals with iSCI has been shown to be more variable than $\mathrm{AB}$ individuals across a variety of overground walking conditions (e.g., walking on a hard and foam surfaces) (Lemay et al., 2020). These increases in the variability of spatial and temporal parameters during walking for individuals with iSCI may be explained by impaired 
sensorimotor function such as muscle weakness and a lack of available afferent information (Day et al., 2011). Adequate afferent input from the central nervous system is required in order to maintain balance during dynamic tasks like walking (Dietz et al., 2002). However, for individuals with iSCI, the flow of sensory information is interrupted and, as a result, the chance of error in the output (i.e., step) increases. These mechanisms may explain why individuals with iSCI have increased stepping variability during walking and in response to an unpredictable perturbation as demonstrated in our study.

In individuals living with stroke and older adults, impaired reactive stepping behaviour has been described as lacking in the magnitude of the response to an unpredictable perturbation. For example, during the Lean-and-Release test, individuals with stroke take steps with low foot clearance and are sometimes unable to generate a stepping response (Mansfield et al., 2012). Older adults have also been shown to take shorter reactive steps compared to younger individuals (Oberg et al., 1993), a finding that conflicts with our results in individuals with iSCI. Instead, our study demonstrated increased variability of the length and width of reactive steps, which, to our knowledge, has not been examined in the stroke or older adult populations.

The results of our study have implications for the development of new rehabilitation training protocols that seek to improve reactive balance. Our findings, along with the study findings of Chan et al. (2020), suggest that the spatial parameters of reactive stepping, rather than the temporal parameters, may be worthwhile targets for balance training. More specifically, training approaches that reduce excessive movement variability may be particularly relevant. One training approach that has been shown to reduce excessive movement variability is repetitive practice. A recent study involving individuals with iSCI found that, after repetitive practice of walking on a treadmill with and without stabilization, gait variability decreased (Wu et al., 
2019). Although the effect of repetitive practice on reducing variability of the spatial parameters of reactive stepping is unknown, a new balance training protocol involving repetitive external perturbations, referred to as perturbation-based balance training, has been developed and was shown to be feasible for use in the ambulatory iSCI population (Unger et al., 2021). Another training approach to consider is strengthening the lower limb muscles involved in reactive stepping. Muscle weakness may contribute to increased stepping errors and increased variability of step length and width (Bogen et al., 2020; Shin et al., 2012). One innovative way to strengthen weak muscles after iSCI is through the application of functional electrical stimulation (FES), which generates artificial muscle contractions using surface electrodes placed on the skin (de Freitas et al., 2018). A recent case study combined lower limb FES with perturbation-based balance training and reported clinically significant improvements on clinical measures of postural control (Gauthier et al., 2020). Based on the findings of the current study, we recommend that measurement of the spatial parameters of reactive stepping is included in future clinical trials targeting reactive postural control.

As indicated in our results, no correlation was found between the variability of the spatial parameters and 3-month fall history. Although unexpected, this finding may be a result of the multi-factorial nature of the causes of falls after SCI. Specifically, ambulatory individuals with SCI experience falls as a result of unique biological, behavioural, and environmental contributing factors (Jørgensen \& Roaldsen, 2017; Singh et al., 2021*, Singh et al. 2020). Further, the 3month fall history was self-reported retrospectively and therefore susceptible to recall bias. Another unexpected finding was the lack of correlation between the variability of the spatial parameters and the behavioural response (i.e., proportion of trials with a single-step or fall responses). Similar to the causes of falls outlined above, a variety of factors likely influence 
performance on the Lean-and-Release test, including a state of anxiety induced by the test's forward fall.

One of the main limitations of this study was a small sample size. This study was a secondary analysis of previously collected data and although 21 participants were recruited, many of the motion capture data sets, critical for the spatial measures used in this study, were lost or faulty. Another limitation is related to generalizability of the results. Our sample had a smaller proportion of individuals with traumatic SCI and fewer males than females, which is different from the Canadian SCI population (Noonan et al., 2012). As a fear of falling is more prevalent in women than men (Pohl et al., 2015), females may have been more motivated to participate in a balance study. Finally, our study was limited by participants' awareness that a release would occur. Although 'sham' trials were included randomly throughout each session, participants may have been able to use anticipatory balance strategies to prepare for a forward fall before the release occurred.

\subsection{Conclusions}

In conclusion, we found greater variability in reactive step length and width for individuals with iSCI, which may contribute to the impaired reactive stepping response observed in this population. Future studies that quantify change in step length and width variability with balance training are warranted. 


\section{References}

Arora, T., Musselman, K. E., Lanovaz, J., Linassi, G., Arnold, C., Milosavljevic, S., Oates, A. (2020). Reactive balance responses to an unexpected slip perturbation in individuals with incomplete spinal cord injury. Clinical Biomechanics, 78, 105099.

Berg, K., Wood-Dauphinee, S., \& Williams, J. I. (1995). The Balance Scale: reliability assessment with elderly residents and patients with an acute stroke. Scandinavian Journal of Rehabilitation Medicine, 27(1), 27-36.

Bogen, B., Moe-Nilssen, R., Aaslund, M. K., \& Ranhoff, A. H. (2020). Muscle strength as a predictor of gait variability after two years in community-living older adults. The Journal of Frailty \& Aging, 9(1), 23-29.

Brotherton, S. S., Krause, J. S., \& Nietert, P. J. (2007). Falls in individuals with incomplete spinal cord injury. Spinal Cord. 45(1), 37-40.

Chan, K., Lee, J. W., Unger, J., Yoo, J., Masani, K., \& Musselman, K. E. (2020). Reactive stepping after a forward fall in people living with incomplete spinal cord injury or disease. Spinal Cord. 58, 185-193.

Day, K. V., Kautz, S. A., Wu, S. S., Suter, S. P., Behrman, A. L. (2011). Foot placement variability as a walking balance mechanism post-spinal cord injury. Clinical Biomechanics, $13,13$.

de Freitas, G. R., Szpoganicz, C., \& Ilha, J. (2018). Does neuromuscular electrical stimulation therapy increase voluntary muscle strength after spinal cord injury? A systematic review. Topics in Spinal Cord Injury Rehabilitation, 24(1), 6-17. 
Dietz, V., Müller, R., \& Colombo, G. (2002). Locomotor activity in spinal man: Significance of afferent input from joint and load receptors. Brain: A Journal of Neurology, 125(Pt 12), 2626-2634.

Do, M. C., Breniere, Y., \& Brenguier, P. (1982). A biomechanical study of balance recovery during the fall forward. Journal of Biomechanics, 15(12), 933-939.

Gauthier, C., Chan, K., Masani, K., Musselman, K. E. (2020). Perturbation-based training in combination with functional electrical stimulation: A promising mixed-methods case study. Archives of Physical Medicine and Rehabilitation, 101(11): (abstract e26).

Hageman, P. A., \& Blanke, D. J. (1986). Comparison of gait of young women and elderly women. Physical Therapy, 66(9), 1382-1387.

Hilliard, M. J., Martinez, K. M., Janssen, I., Edwards, B., Mille, M.-L., Zhang, Y., \& Rogers, M. W. (2008). Lateral balance factors predict future falls in community-living older adults. Archives of Physical Medicine and Rehabilitation, 89(9), 1708-1713.

Hof, A. L., Gazendam, M. G., \& Sinke, W. E. (2005). The condition for dynamic stability. Journal of biomechanics, 38(1), 1-8.

Hsiao-Wecksler, E. T., \& Robinovitch, S. N. (2007). The effect of step length on young and elderly women's ability to recover balance. Clinical Biomechanics (Bristol, Avon), 22(5), 574-580.

Jørgensen, V., \& Roaldsen, K. S. (2017). Negotiating identity and self-image: perceptions of falls in ambulatory individuals with spinal cord injury - a qualitative study. Clinical Rehabilitation, 31(4), 544-554. 
Jørgensen, V., Butler Forslund, E., Opheim, A., Franzén, E., Wahman, K., Hultling, C., Seiger, Å., Ståhle, A., Stanghelle, J. K., \& Roaldsen, K. S. (2017). Falls and fear of falling predict future falls and related injuries in ambulatory individuals with spinal cord injury: a longitudinal observational study. Journal of Physiotherapy, 63(2), 108-113.

Khan, A., Pujol, C., Laylor, M., Unic, N., Pakosh, M., Dawe, J., \& Musselman, K. E. (2019). Falls after spinal cord injury: A systematic review and meta-analysis of incidence proportion and contributing factors. Spinal Cord. 57(7), 526-539.

Lemay, J. F., Noamani, A., Unger, J., Houston, D. J., Rouhani, H., Musselman, K. E. (2020). Using wearable sensors to characterize gait after spinal cord injury: Evaluation of testretest reliability and construct validity. Spinal Cord, E-pub Oct 6.

Maki, B. E., \& Mcilroy, W. E. (2006). Control of rapid limb movements for balance recovery: Age-related changes and implications for fall prevention. Age Ageing, 35(2), ii12-ii18.

Mansfield, A., Inness, E. L., Lakhani, B., \& McIlroy, W. E. (2012). Determinants of limb preference for initiating compensatory stepping post- stroke. Archives of Physical Medicine and Rehabilitation, 93(7), 1179-1184.

Mansfield, A., Inness, E. L., Wong, J. S., Fraser, J. E., \& McIlroy, W. E. (2013). Is impaired control of reactive stepping related to falls during inpatient stroke rehabilitation? Neurorehabilitation and Neural Repair, 27(6), 526-533.

Martinez, K. M., Rogers, M. W., Blackinton, M. T., Cheng, M. S., \& Mille, M. L. (2019). Perturbation-induced stepping post-stroke: A pilot study demonstrating altered strategies of both legs. Frontiers in Neurology, 10, 711. 
McCrum, C., Gerards, M. H. G., Karamanidis, K., Zijlstra, W., \& Meijer, K. (2017). A systematic review of gait perturbation paradigms for improving reactive stepping responses and falls risk among healthy older adults. European Review of Aging and Physical Activity, 14(1).

Medell, J. L., \& Alexander, N. B. (2000). A clinical measure of maximal and rapid stepping in older women. The Journals of Gerontology Series A: Biological Sciences and Medical Sciences, 55(8), M429-M433.

Musselman, K. E., Arnold, C., Pujol, C., Lynd, K., \& Oosman, S. (2018). Falls, mobility and physical activity after spinal cord injury: An exploratory study using photo-elicitation interviewing. Spinal Cord Series and Cases. 4, 39.

Noonan, V. K., Fingas, M., Farry, A., Baxter, D., Singh, A., Fehlings, M. G., \& Dvorak, M. F. (2012). Incidence and prevalence of spinal cord injury in Canada: A national perspective. Neuroepidemiology, 38(4), 219-226.

Oberg, T., Karsznia, A., \& Oberg, K. (1993). Basic gait parameters: reference data for normal subjects, 10-79 years of age. Journal of Rehabilitation Research and Development, 30(2), $210-223$.

Pohl, P., Ahlgren, C., Nordin, E., Lundquist, A., \& Lundin-Olsson, L. (2015). Gender perspective on fear of falling using the classification of functioning as the model. Disability and Rehabilitation, 37(3), 214-222.

Shin, S., Valentine, R. J., Evans, E. M., \& Sosnoff, J. J. (2012). Lower extremity muscle quality and gait variability in older adults. Age and Ageing, 41(5), 595-599. 
Singh, H., Chan, K., Cheung, L., Hitzig, S., Musselman, K.E. (2021*). The impact of falls and fear of falling on participation, autonomy, and life satisfaction among individuals with spinal cord injury: a brief report. Journal of Spinal Cord Medicine, Under Review.

Singh, H., Shibi Rosen, A., Bostick, G., Kaiser, A., \& Musselman, K. E. (2020). Exploring the causes and impacts of falls among ambulators with spinal cord injury using photovoice: A mixed methods study. BMJ Open. 10, e039763.

Unger, J., Chan, K., Lee, J. W., Craven, B. C., Mansfield, A., Alavinia, M., Masani, K., \& Musselman, K. E. (2021). The effect of Perturbation-based Balance Training and Conventional Intensive Balance Training on reactive stepping ability in individuals with incomplete spinal cord injury or disease: A randomized clinical trial. Frontiers in Neurology, 12, 620367.

Unger, J., Oates, A. R., Lanovaz, J., Chan, K., Lee, J. W., Theventhiran, P., Masani, K., \& Musselman, K. E. (2020). The measurement properties of the Lean-and-Release test in people with incomplete spinal cord injury or disease. The Journal of Spinal Cord Medicine, $1-10$.

Weaver, T. B. (2017). New insights into the landing phase of reactive stepping: Predictors of control, muscle recruitment, movement restraints and two-step responses. Doctoral dissertation, University of Waterloo, Waterloo Canada.

World Health Organization. Falls fact sheet. (2018). https://http://www.who.int/mediacentre/factsheets/fs344/en. 
Wu, M. M., Brown, G. L., Kim, K. A., Kim, J., \& Gordon, K. E. (2019). Gait variability following abrupt removal of external stabilization decreases with practice in incomplete spinal cord injury but increases in non-impaired individuals. Journal of Neuroengineering and Rehabilitation, 16(1), 4. 


\section{Chapter 4}

\section{Evaluating the Spatial Measurement Properties of the Lean-and-Release}

\section{Test for Individuals with Incomplete Spinal Cord Injuries}

\subsection{Introduction}

In order to prevent a fall after an unexpected perturbation, one must produce an effective reactive balance response (Maki \& Mcilroy, 2006). These responses often involve taking a reactive step, which acts to increase an individual's base of support $(\mathrm{BoS})$ such that the position of their centre of mass (CoM) remains within it (Hilliard et al., 2008; Mansfield et al., 2012; Mansfield et al., 2013; McCrum et al., 2017). When a single step does not adequately adjust the position of one's CoM within their BoS, additional steps may be taken although this strategy is not ideal (Wolfson et al., 1986). Recent studies have demonstrated that individuals with incomplete spinal cord injury (iSCI) are more likely to require multiple reactive steps compared to able-bodied individuals (Chan et al., 2020). Additionally, people with iSCI take reactive steps that are more variable in length and width than their AB counterparts (Heffernan et al., 2021*). Impairments in reactive stepping ability are also evident in other clinical populations. Specifically, individuals with stroke have demonstrated deficiencies in reactive stepping ability, such as reduced foot clearance, which have been directly linked to an increased risk of falling (Mansfield et al., 2013).

A standardized approach to evaluate and quantify reactive stepping is the Lean-and-Release test (Inness et al., 2015). This test, which simulates a forward fall from a standing position, involves participants leaning forward while being supported by a horizonal cable attached to the back of a harness at waist height (figure 1). As the participant leans forward, their body weight is 
supported by the cable until it is released unpredictably, which initiates a forward fall requiring a reactive stepping response. During the test, a number of reactive stepping parameters can be measured. Behavioural responses (i.e., the number of steps taken) can be recorded through observation. More technical characteristics of the reactive stepping response like the temporal (i.e., foot off, foot contact, and swing time) and spatial (i.e., step length and width) parameters are recorded using force plate and motion capture systems. A growing number of studies have used the Lean-and-Release test to evaluate reactive stepping in people with stroke (Inness et al., 2015; Mansfield et al., 2011) and iSCI (Chan et al., 2020) and recently it has been used to assess the effectiveness of balance training interventions for these individuals (Mansfield et al., 2018; Unger et al., 2021). Therefore, it is important to understand the reliability and validity of the Lean-and-Release test in order to accurately attribute any change in performance on the Leanand-Release test with a true change in balance ability after a given intervention.

When evaluating a measurement's reliability, we consider the degree to which it produces a consistent value each time it is used with the assumption that no actual change in performance as occurred (Streiner et al., 2015). The test-retest reliability of a measurement represents how well a test can reproduce the same performance values over time when the test subject's status has not changed in a way that would influence their performance (Mokkink et al., 2010). Additionally, reliability can be assessed by considering the agreement a measurement has by using measurement error to analyse the degree of accuracy it has when the measurement is repeated (de Vet et al., 2006). When evaluating a measurement's validity, we consider the degree to which the test measures what it sets out to measure (Streiner et al., 2015). More specifically, a test's convergent validity demonstrates the degree to which the outcome measure of interest relates to another outcome measure known to measure a similar paradigm (Streiner et al., 2015). 
In the iSCI population, the reliability and validity of the behavioural and temporal parameters of the Lean-and-Release test have been examined (Unger et al., 2020). The results of the study by Unger et al. (2020), demonstrated that the behavioural response of the Lean-and-Release test had good test-retest reliability and convergent validity (i.e. significant positive correlations with lower extremity strength scores and self-reported balance confidence) (Unger et al., 2020). However, only one of the temporal parameters (foot contact) was found to have good test-retest reliability. To date, the reliability and validity of the spatial parameters of the Lean and Release test have not been established; yet the spatial parameters of reactive stepping are probable targets for balance training and assessment in people with iSCI (Heffernan et al., 2021*). Hence, the aim of this study was to assess the test-retest reliability, agreement, and construct validity of the Lean-and-Release test's spatial parameters (i.e., step length, step width, step length variability, step width variability) for individuals with iSCI. We hypothesized that mean step length and width measurements derived from the Lean-and-Release test would have a clinically useful degree of test-retest reliability (i.e. intra-class correlation coefficients (ICC) $\geq 0.70$ ), but that step length variability and step width variability would have a poor degree of test-retest reliability (i.e. ICC $\leq 0.50$ ). This hypothesis is consistent with previous research that evaluated the testretest reliability of wearable sensors to measure stride length during walking for people with iSCI (Lemay et al., 2021). Based on the results of the validation of the behavioural and temporal components of reactive stepping (Unger et al. 2020), we also hypothesized that the spatial parameters measured during the Lean-and-Release test would demonstrate convergent validity as evidenced by moderately strong correlations with lower extremity strength and performance on other balance measures. 


\subsection{Methods}

The University Health Network Research Ethics Board granted ethical approval for this study. Experimental procedures were conducted at the Lyndhurst Centre, Toronto Rehabilitation Institute, University Health Network. The data analysed for this study were collected as part of a clinical trial that tested the effects of perturbation-based balance training on balance ability in participants with iSCI (ClinicalTrials.gov identifier NCT02960178) (Unger et al., 2019, 2021).

\subsubsection{Participants}

Participants were recruited using a central recruitment database which contained the names of people with iSCI. These individuals were inpatients at the Lyndhurst Centre who had consented to being contacted regarding ongoing research studies. Additionally, individuals with iSCI were able to inquire about participating through information posted on recruitment posters at the Lyndhurst Centre.

Individuals were eligible for inclusion in the study if they met the following criteria: 1) were at least 18 years old; 2) diagnosed with a chronic ( >1 year post-injury), traumatic or non-traumatic and non-progressive motor iSCI; 3) a level C or D injury as defined by the American Spinal Injury Association Impairment Scale (AIS); 4) demonstrated ability to reach forward with arms at least 2 inches in an unsupported standing position (moderate level of trunk control according to the Berg Balance Scale) (Berg et al., 1995); 5) demonstrated ability to stand independently for 30 seconds; and 6) free of any other conditions affecting balance and/or walking ability. Individuals were excluded from participation in the study if they had any of the following: 1) inability to achieve a standing position due to contractures or spasticity; 2) a pressure injury on 
the pelvis or trunk (grade 2 or greater); and/or 3) a history of fragility fracture(s) in the lower extremities.

\subsubsection{Data Acquisition}

Participants included in our study completed two testing sessions spaced two weeks apart, each involving the Lean-and-Release test. Before participating in testing sessions, the age and sex of each eligible participant were collected. Additional data collected for our study included participant's neurological level of injury and time post-injury.

As illustrated in figure 3.1, participants donned a safety harness connected to a ceiling attachment during the Lean-and-Release test. This system provided support for participants in the event of a fall. A horizontal cable leading to a force transducer and release mechanism was also attached to the back of the participant's safety harness at waist height. To start each trial, participants stood on a dual force plate system consisting of four $25.1 \times 50.2 \mathrm{~cm}$ components (AccuSway Dual, Advanced Mechanical Technology Inc., Watertown, USA). A researcher ensured each of the participant's feet were placed on one of the two force plate components closest to the release mechanism. From this position, a researcher instructed participants to lean forward while maintaining hip extension. Once the researcher was able to confirm that $8-12 \%$ of the participants body weight was being supported by the cable, it was manually released within 30 seconds. By releasing the cable at the $8-12 \%$ target, a fall in the anterior direction is initiated requiring a reactive step response in order to maintain balance (Mansfield et al., 2013; Unger et al., 2020). Each session consisted of up to 10 Lean-and-Release trials. In an effort to diminish anticipatory responses, three trials in which participants leaned forward for 30 seconds but no release occurred were also included randomly (i.e. sham trials). To ensure consistency in testing conditions between the two sessions, instructions for the Lean-and-Release test were 
standardized. Additionally, the researchers were trained to administer the testing protocol and remained consistent for each participant for both sessions. A licensed therapist was also present for each testing session.

The force plate system used during the Lean-and-Release trials recorded kinetic data during the testing sessions. These data were then filtered using a 4th order low-pass Butterworth filter with a cut-off frequency of $30 \mathrm{~Hz}$. Kinetic data collected using the force plates were used to identify important temporal parameters of the reactive step such as when a reactive step began (foot-off time) and when a reactive step ended (foot contact time). A 3D motion capture system was used to collect the kinematic data for this study. The system consisted of six cameras (Rapter-E; Motion Analysis Corp., USA) and 35 reflective markers secured to anatomical landmarks of the participants. The two markers used for the purposes of this study were attached to the participant's shoes at each heel. Using a sampling frequency of $200 \mathrm{~Hz}$ (Cortex ver. 3.1, Motion Analysis Corp., USA) motion capture data collected during Lean-and-Release trials determined the spatial parameters (i.e. length and width) of the first reactive step.

During the first testing session, gait parameters (i.e. step length and step width) were also collected by the research team using a pressure sensitive mat (Zeno Walkway, Model 485, Ver. J; Prokinetics, Havertown Pennsylvania, USA). Participants walked across the mat at a comfortable pace without using a gait aid, if feasible. Also at the first testing session, a licensed physical therapist evaluated participants' balance abilities using the Mini-Balance Evaluations Systems Test (mini-BESTest) (Franchignoni et al., 2010) and balance confidence using the Activities-specific Balance Confidence Scale (ABC Scale) (Powell \& Myers, 1995). Both scales are valid and reliable measures for individuals with chronic iSCI (Chan et al., 2019; Jørgensen et al., 2017; Shah et al., 2017). The mini-BESTest evaluates four components of postural control 
(i.e. reactive postural control, anticipatory, sensory orientation, and dynamic gait) with a maximum possible score of 28 points. The ABC scale involves reporting one's level of confidence to maintain balance during 16 different standing and walking tasks on a scale of $0 \%$ $100 \%$, with $100 \%$ representing high balance confidence. Finally, lower extremity (LE) strength was also evaluated during the first testing session. A licensed physical therapist used manual muscle testing (MMT) to evaluate the strength of 12 muscle groups of each leg (hip flexors, hip extensors, hip abductors, hip adductors, hip internal rotators, hip external rotators, knee flexors, knee extensors, ankle internal rotators, ankle external rotators, ankle plantar flexors, and ankle dorsiflexors). MMT is considered a valid measure of muscle strength for people with SCI (Schwartz et al., 1992). Participants performed full range of motion contractions against manual resistance provided by the therapist, who assigned a score for each muscle group on an ordinal scale of 0-5, where a higher score indicates a higher degree of strength (Kendall \& Al, 2010). A total LE MMT score was calculated by summing the scores of the individual muscle groups of both legs. 


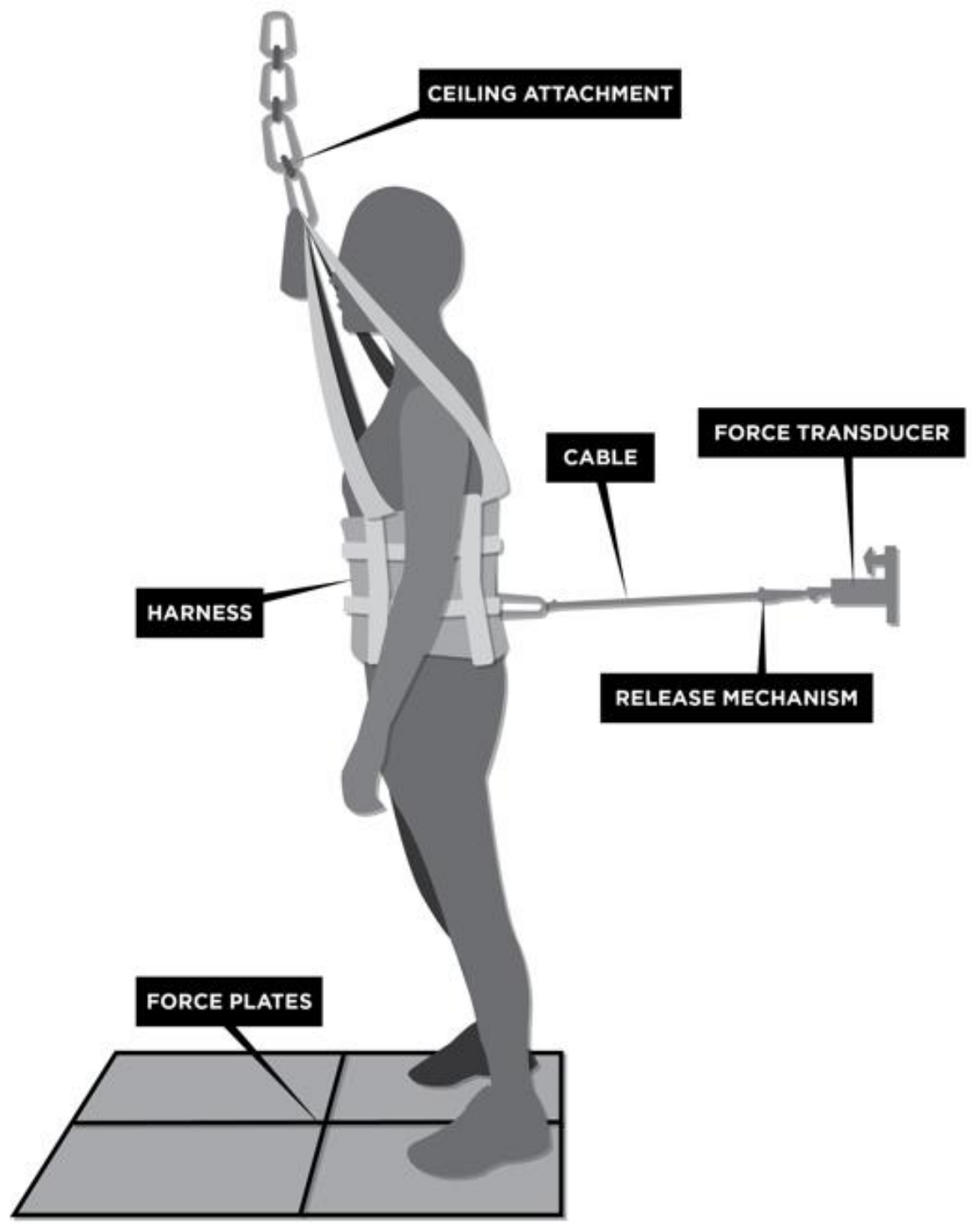

Figure 3.1. Configuration of the Lean-and-Release test (Chan et al., 2020). Copyright permission granted.

\subsubsection{Data Analysis}

The age and time post-injury of each participant were reported as a mean value $( \pm 1$ standard deviation) and a frequency count was used to report sex. A computational software (MATLAB 2018a; MathWorks, USA) running a custom-written script was used to process the kinematic and kinetic data collected for this study. A reactive step was determined to have started when the 
force plate in contact with the stepping foot registered $<1 \%$ of the participant's body weight (i.e. foot-off time). The end of a reactive step occurred when the force plate contacted by the stepping foot registered $>1 \%$ of the participant's body weight (i.e. foot contact time). Figure 3.2 illustrates the measurement of spatial parameters during the Lean-and-Release trials. Step length and width were calculated by measuring change in position (centimetres) of the heel marker of the stepping foot during the first reactive step (time of foot-off to time of foot contact) where step length represented change in the anterior-posterior direction and step width represented change in the medial-lateral direction. A narrowing of the BoS after a reactive step resulted in negative step width value. These methods for calculating step length and width have been used in previous studies examining the spatial characteristics of reactive stepping in older adults and individuals with stroke (Martinez et al., 2019; Weaver, 2017).

The mean ( \pm 1 standard deviation) step length and width of the first reactive steps taken during a testing session were calculated for each participant. Additionally, the variability of step length and width was represented by the calculated standard deviation across reactive stepping trials in a testing session for each participant.

The test-retest reliability of the spatial parameters of the Lean-and-Release test (i.e. mean step length, mean step width, step length variability, and step width variability) was evaluated using an ICC (two-way mixed effects model, absolute agreement) and data from the first and second testing sessions. The ICC is an appropriate measure of test-retest reliability and helps determine degree of reliability by interpreting the value as poor $(<0.50)$, moderate $(0.50-0.75)$, good $(0.75-$ 0.90), or excellent (>0.90) (Koo \& Li, 2016). For the purposes of this study, an ICC value of 0.70 or greater was considered a clinically useful degree of reliability (Streiner et al., 2015). Bland-Altman plots were created for each of the spatial parameters to examine the agreement 
between the first and second testing sessions. With limits of agreement set to 95\%, the difference between the two sessions for a given spatial parameter was plotted on the y-axis and the mean value of that spatial parameter plotted on the $\mathrm{x}$-axis. Convergent validity was evaluated by examining the correlations between the spatial parameters of the Lean-and-Release test collected at the first session and performance on the following related measures: 1) gait parameters (i.e. gait stride length and gait stride width), 2) mini-BESTest score (i.e. total score and reactive postural control subscale score), 3) ABC Scale score, 4) total LE MMT score (i.e. sum of 24 muscles, 12 per leg), and 5) LE MMT scores for specific muscle groups known to be involved in generating a reactive step, namely hip flexors, knee flexors, knee extensors, ankle plantar flexors and ankle dorsiflexors (Chan et al., 2020). Since participants used both their right and leg lefts to take reactive steps, the LE MMT score for each muscle group was a sum of the scores for the right and left legs. These data sets were tested for normality using a Shapiro-Wilk test and for equality of variances using a Levene's test. Based on the results of these tests, Pearson's R or Spearman's rho were used to determine correlation coefficients which were considered strong (0.90-1.00), moderately strong $(0.70-0.90)$, moderate $(0.50-0.70)$, or low $(0.30-0.50)$ (Mukaka, 2012). SPSS statistical analysis software (IBM, Armonk, NY, USA) was used to analyze the data in this study and alpha was set to 0.05 . 
Length

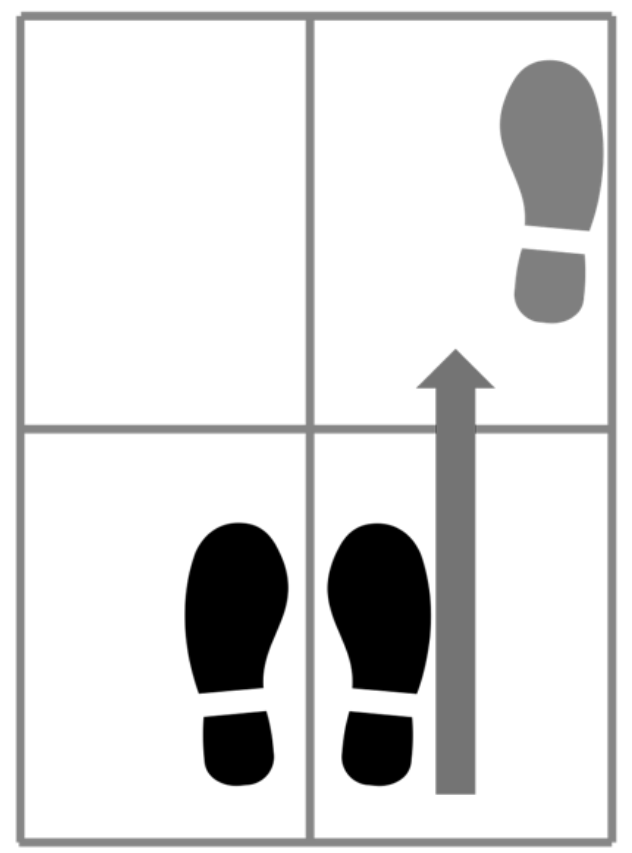

Width

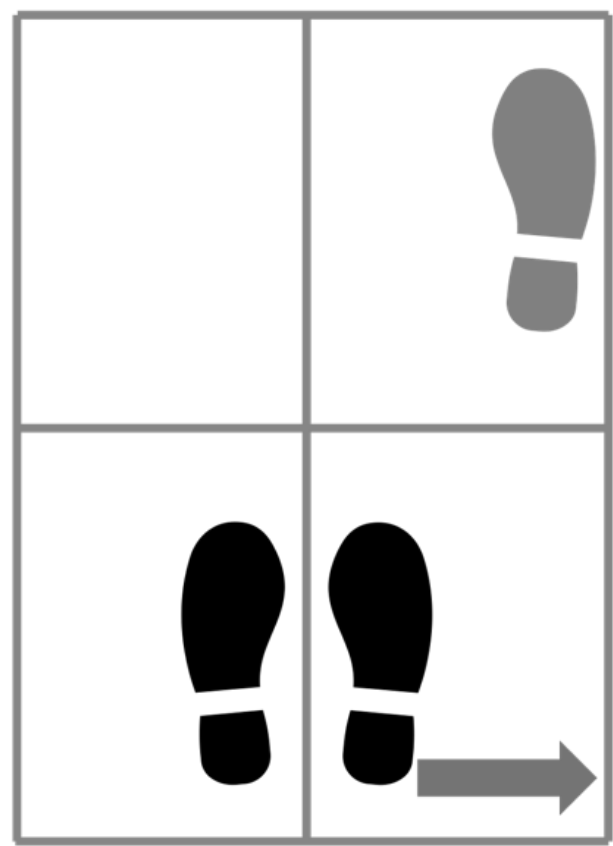

Figure 3.2. Spatial characteristics of reactive stepping measured using starting heel marker position at foot-off time (black footprint) and heel marker position after first step at foot contact time (grey footprint). Arrows represent direction of measurement. Rectangles represent force plate configuration.

\subsection{Results}

\subsubsection{Participants}

Twenty-one individuals met the inclusion criteria and participated in our study. However, some participants were not able to perform any reactive steps during the Lean-and-Release trials $(n=4)$ and some testing sessions had motion capture and/or force plate data that were missing or faulty $(\mathrm{n}=6)$. As a result, 10 participants ( 8 females, 2 males $)$ were included in this analysis with a mean age of 52.3 (16.0) years. During the first testing session, five of the participants were able to complete ten trials, one participant completed nine trials, one participant completed eight trials, two participants completed seven trials and one participant completed six trials of the Lean-andRelease test. During the second testing session five of the participants were able to complete ten 
trials, four participants completed nine trials, and one participant completed six trials. The demographic data of the participants included in our analyses are reported in Table 4.1.

Table 4.1. Demographic and injury data for participants.

\begin{tabular}{ccccc}
\hline ID \# & Sex & $\begin{array}{c}\text { Age } \\
\text { (years) }\end{array}$ & $\begin{array}{c}\text { Time post-injury } \\
\text { (years) }\end{array}$ & Level of injury \\
\hline PBT 5 & F & 32 & 3.5 & C4 \\
PBT 8 & M & 60 & 3.2 & C5 \\
PBT 10 & F & 43 & 3.9 & T6 \\
PBT 14 & F & 59 & 1.1 & C1 \\
PBT 16 & F & 55 & 9.1 & C5 \\
PBT 17 & F & 38 & 1.3 & T4 \\
PBT 18 & F & 54 & 13 & C4 \\
PBT 20 & F & 56 & 1.2 & L5 \\
PBT 22 & M & 88 & 5.3 & T11 \\
PBT 23 & F & 38 & 6.8 & - \\
\hline Mean & & 52.3 & 4.8 & - \\
(SD) & - & $(16.0)$ & $(3.8)$ & - \\
\hline Count & 2M & - & & \\
\hline
\end{tabular}

\subsubsection{Reliability}

Mean step length recorded during the Lean-and-Release test demonstrated good test-retest reliability ( $\mathrm{ICC}=0.90$, C.I.: 0.62-0.97). Mean step width recorded during the Lean-and-Release test also demonstrated good test-retest reliability ( $\mathrm{ICC}=0.76$, C.I.: 0.09-0.94). However, a poor degree of test-retest reliability was indicated by the ICCs for step length variability (ICC $=0.04$, C.I.: $-1.70-0.73)$ and step width variability $(\mathrm{ICC}=0.07$, C.I.: $-3.66-0.78)$. 


\subsubsection{Agreement}

The Bland-Altman plots of all spatial parameters of the Lean-and-Release test are shown in figure 4.1. The line of bias (lower limit of agreement, upper limit of agreement) for the spatial parameters of the Lean-and-Release test were as follows: mean step length $-1.88(-10.60,6.82)$, mean step width $0.78(-4.40,5.95)$, step length variability $1.90(-5.31,9.12)$, and step width variability $0.43(-4.13,4.98)$. Visual analysis of the Bland-Altman plots indicates that the biases are small and not clinically relevant; however, the limits of agreement are wide.
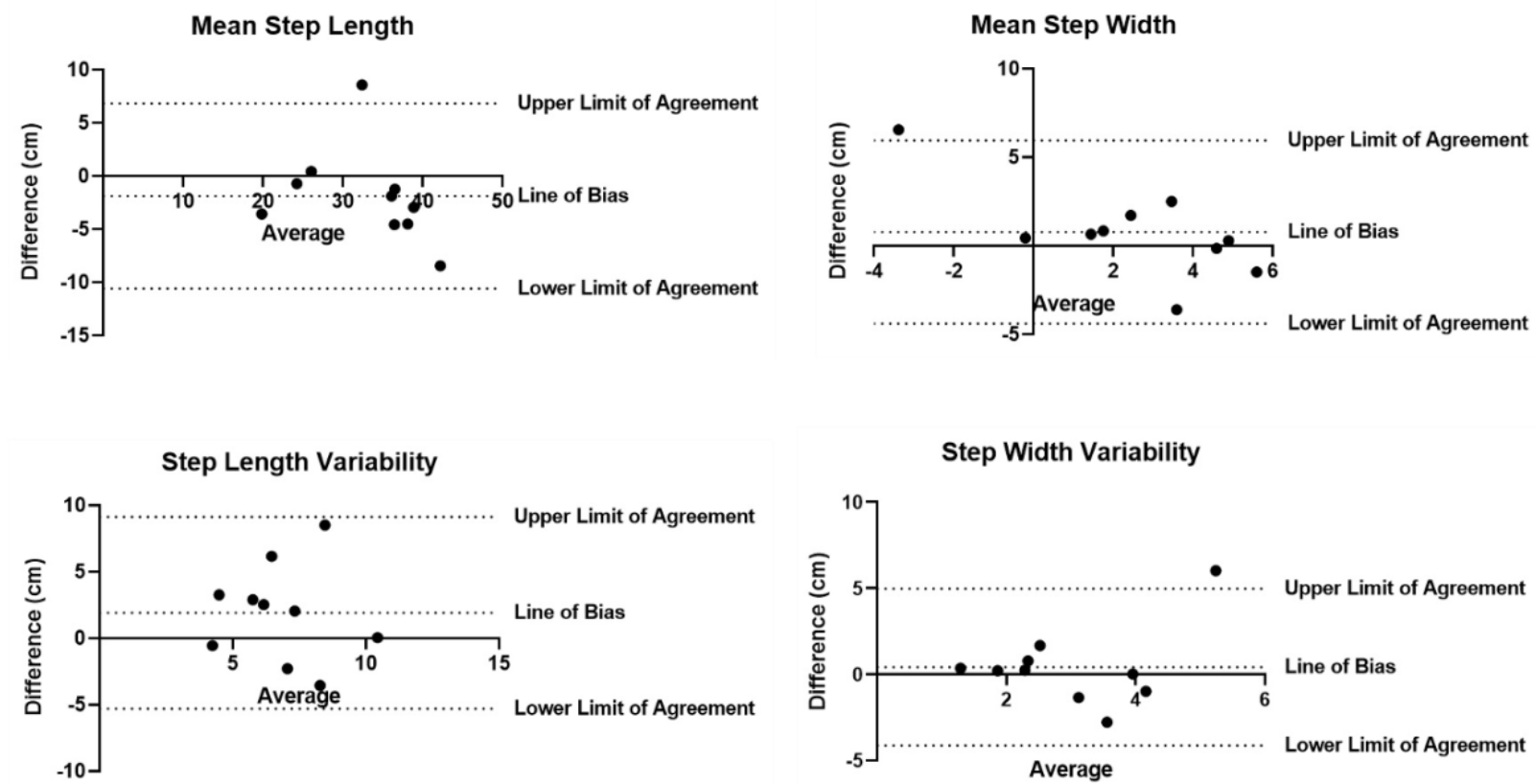

Figure 4.1. Bland-Altman plots showing agreement between first and second Lean-andRelease testing sessions for each spatial parameter. Limits of agreement (dashed horizontal lines) are set to the $95 \%$ confidence interval. Line of bias indicated by solid horizontal line. 


\subsubsection{Convergent Validity}

Mean step length recorded during the Lean-and-Release test was found to have the following significant correlations (see Table 4.2): 1) a moderately strong, positive correlation with stride width recorded during the gait assessment $(\mathrm{r}(8)=0.80, \mathrm{p}=0.01), 2)$ a moderately strong, negative correlation with the reactive postural control subscale score of the mini-BESTest $(r(8)=$ $-0.65, \mathrm{p}=0.04), 3$ ) a moderately strong, negative correlation with the total score of the LE MMT $(\mathrm{r}(8)=-0.66, \mathrm{p}=0.04) 4)$ a moderately strong, negative correlation with hip flexion strength $(\mathrm{r}(8)=-0.89, \mathrm{p}=0.01)$, and 5) a moderately strong, negative correlation with knee flexion strength $\left(\mathrm{r}_{\mathrm{s}}(8)=-0.70, \mathrm{p}=0.02\right)$. There were no significant correlations between the mean step width recorded during the Lean-and-Release test and performance on measures of gait, balance, balance confidence or LE strength.

Significant correlations were also found between measures of variability in the spatial parameters of reactive stepping during the Lean-and-Release test and measures of LE strength (see Table 2). Specifically, step length variability demonstrated a moderately strong, negative correlation with the ankle dorsiflexion strength $(\mathrm{r}(8)=-0.69, \mathrm{p}=0.03)$, and step width variability demonstrated a moderately strong, negative correlation with the knee flexion strength $\left(\mathrm{r}_{\mathrm{s}}(8)=-0.68, \mathrm{p}=0.03\right)$. 
Table 4.2. Convergent validity.

\begin{tabular}{|c|c|c|c|c|c|c|c|c|c|}
\hline & & $\begin{array}{c}\text { Mean Step } \\
\text { Length }\end{array}$ & $\boldsymbol{P}$ & $\begin{array}{c}\text { Mean Step } \\
\text { Width }\end{array}$ & $P$ & $\begin{array}{c}\text { Step Length } \\
\text { Variability }\end{array}$ & $\boldsymbol{P}$ & $\begin{array}{l}\text { Step Width } \\
\text { Variability* }\end{array}$ & $P$ \\
\hline \multirow{2}{*}{ Gait } & Stride Length & -0.38 & 0.27 & -0.39 & 0.26 & -0.18 & 0.61 & -0.56 & 0.09 \\
\hline & Stride Width & 0.80 & 0.01 & 0.01 & 0.99 & 0.56 & 0.09 & 0.36 & 0.31 \\
\hline \multirow{2}{*}{ Mini-BESTes } & Total Score & -0.60 & 0.67 & -0.33 & 0.36 & -0.60 & 0.07 & -0.56 & 0.09 \\
\hline & Reactive & -0.65 & 0.04 & -0.45 & 0.19 & -0.57 & 0.09 & -0.60 & 0.07 \\
\hline \multicolumn{2}{|c|}{ ABC Scale } & 0.09 & 0.80 & -0.35 & 0.31 & -0.25 & 0.48 & 0.11 & 0.76 \\
\hline \multirow{6}{*}{ LE MMT } & Total Score & -0.66 & 0.04 & -0.12 & 0.73 & -0.55 & 0.10 & -0.40 & 0.25 \\
\hline & Hip Flexion & -0.89 & 0.01 & 0.06 & 0.87 & -0.29 & 0.41 & -0.55 & 0.10 \\
\hline & Knee Flexion* & -0.70 & 0.02 & -0.24 & 0.51 & -0.25 & 0.48 & -0.68 & 0.03 \\
\hline & Knee Extension & -0.50 & 0.14 & 0.33 & 0.35 & -0.01 & 0.99 & -0.60 & 0.07 \\
\hline & Ankle Dorsiflexion & -0.62 & 0.06 & -0.21 & 0.56 & -0.69 & 0.03 & -0.43 & 0.21 \\
\hline & Ankle Plantarflexion & -0.51 & 0.14 & -0.20 & 0.57 & -0.52 & 0.12 & -0.39 & 0.26 \\
\hline
\end{tabular}

Correlation coefficients (Pearson or *Spearman) with accompanying $P$ value.

Statistically significant results indicated by bold font.

LE MMT total score and score of muscle groups represent summed score of both legs.

\subsection{Discussion}

Here we evaluated the test-retest reliability and convergent validity of the spatial parameters of reactive stepping, as measured with the Lean-and-Release test, in individuals with chronic iSCI. As hypothesized, the mean step length and mean step width of reactive stepping had clinically useful degrees of test-retest reliability, whereas the test-retest reliability of step length variability and step width variability was poor. Our hypothesis concerning the convergent validity of the spatial parameters was partially met; mean step length was found to have convergent validity. Moderately strong to strong correlations were observed between mean step length and the 
following variables: stride width during walking, the score on the reactive subscale of the miniBESTest, LE MMT total score, and hip flexor and knee flexor strength. Interesting, several of these significant correlations were negative, suggesting a greater mean step length was associated with decreased strength and lower scores on the reactive subscale of the mini-BESTest.

Convergent validity of the mean step width of reactive stepping was not observed, as this spatial parameter did not correlate with the other measures of balance, gait, balance confidence or lower extremity strength. Step length variability and step width variability were each found to be associated with the strength of a single muscle group (i.e. ankle dorsiflexors and knee flexors, respectively). Altogether, the findings reported here suggest that the mean step length of the Lean-and-Release test has adequate reliability and validity for clinical use (Streiner et al., 2015); however, the small sample size precludes making firm conclusions.

A previous study examining the psychometric properties of the Lean-and-Release test for people with iSCI demonstrated it to be a reliable measure of the behavioural response and one temporal parameter (i.e. foot contact) of reactive stepping (Unger et al., 2020). Our study adds to this prior research by showing that the test-retest reliability of the spatial parameters measured during the Lean-and-Release test were high for the mean values, but low for the measures of variability. These findings are consistent with the results of another study examining the psychometric properties of wearable sensors to characterize gait parameters in people with iSCI (Lemay et al., 2020). During the study by Lemay and colleagues (2020), wearable sensors were used to measure parameters of gait such as stride length and stride velocity during a variety of walking conditions. Similar to the results of our psychometric evaluation of the Lean-and-Release test, the wearable sensors demonstrated high degrees of test-retest reliability for the mean stride length and velocity measurements, but low degrees of test-retest reliability for the measurements of stride length and velocity variability (Lemay et al., 2020). Research on gait variability has 
demonstrated a threshold for the minimum number of strides in a testing session required to achieve an adequate degree of reliability (Hollman et al., 2010). A similar threshold may also exist when assessing the reliability of reactive step length and width variability. Specifically, in our study, a greater degree of test-retest reliability of step length and width variability may have been achieved if more Lean-and-Release trials were included during the testing session. However, increasing the number of Lean-and-Release trials may not be feasible for the iSCI population since a number of the participants included in our study were not able to complete all 10 trials of the testing session.

A number of related clinical measures were found to correlate with the mean step length of reactive stepping as measured with the Lean-and-Release test. Mean step length was found to negatively correlate with scores on the mini-BESTest's reactive subscale, indicating that a longer mean reactive step is associated with a lower degree of reactive postural control. This finding was somewhat unexpected as prior research has demonstrated that older adults have a shorter reactive step length in comparison to their younger peers (Hsiao-Wecksler \& Robinovitch, 2007; Medell \& Alexander, 2000) who would have greater postural control. Similarly, we also observed significant negative correlations between the mean length of reactive steps and strength measures of the legs, suggesting that a longer mean reactive step is associated with less strength. Although a longer reactive step may seem beneficial as it suggests a greater margin of stability $(\mathrm{MoS})$ (i.e., the distance between the outer boundaries of one's BoS and their extrapolated CoM) (Hof et al., 2005), taking a long reactive step could reduce stability. Increasing the length of the reactive step is likely to increase the moment around one's CoM thereby increasing one's momentum and reducing stability (Yamaguchia \& Masani, 2021*). Hence, participants in this study with lower leg strength may have been unable to precisely control the foot contact of their 
reactive steps and overcompensated by taking a long step that unnecessarily increased momentum and reduced stability.

The mean step length of reactive stepping was also found to have a moderately strong positive correlation with stride width during gait. Although the mechanisms governing reactive stepping and normal walking may differ, this finding suggests those who take wider steps while walking are likely to also take longer reactive steps. This finding also suggests that longer reactive steps may not indicate greater reactive stepping ability.

A prior study comparing the spatial parameters of reactive stepping between people with iSCI and able-bodied individuals demonstrated significantly greater variability in the length and width of the first reactive step for people with iSCI (Heffernan et al., 2021*). These results may help explain the negative correlations of step length variability and step width variability, as measured during the Lean-and-Release test, with specific LE MMT scores (i.e. for ankle dorsiflexion and knee flexion, respectively). For example, an individual with poor strength of the leg muscles may not have adequate and consistent control of the stepping foot and as a result take reactive steps that are more variable in length and width.

Mean step width, as measured with the Lean-and-Release test, did not correlate with any clinical measures of balance, gait, balance confidence or LE strength. This finding may be explained by the nature of the perturbation created by the Lean-and-Release test. This test elicits a perturbation in the anterior-posterior direction, and hence, necessitates little change in the BoS in the mediolateral direction.

To the best of our knowledge, this is the first study to assess the psychometric properties of the spatial parameters of the Lean-and-Release test in a patient population. However, previous 
research has examined the reliability of the spatial parameters derived from the Lean-andRelease test for $\mathrm{AB}$ adults across a wide age range (21-80 years). The study by Werth et al. (2021), also tested two perturbation variations of the Lean-and-Release test; maximal lean angle (maximum $\%$ body mass supported in forward lean that required just one step after release) and pre-determined lean angle (21-25\% body mass supported in forward lean). The results of the study indicate that spatial parameters of reactive stepping ( $\mathrm{MoS}$ and $\mathrm{BoS}$ at foot contact) measured with the Lean-and-Release test are reliable for $\mathrm{AB}$ adults independent of the lean angle condition (Werth et al., 2021). Although the percentage of an individual's body weight supported in forward lean required to initiate a reactive step may differ between clinical populations and $\mathrm{AB}$ individuals, these results could be translated to support the use of the Lean-and-Release test as an assessment tool for people with iSCI. Specifically, a less technical measurement of lean before release could make the Lean-and-Release test more accessible for use in clinical settings.

This study was limited by a small sample size. Although twenty-one participants were recruited for the study, a significant number of data sets $(n=6)$ were lost or faulty and therefore unavailable for analysis. Unfortunately, because this was a secondary analysis of previously collected data, we were unable to recruit additional participants to account for lost/faulty data sets. Our study group was also not representative of the proportion of males to females in the Canadian SCI population where males are more likely to have a SCI (Noonan et al., 2012). Instead, our study group consisted of two males and eight females which may limit the generalizability of our results. Although MMT is considered a valid measure of muscle strength for people with SCI (Schwartz et al., 1992), other measures may provide a more definitive understanding of muscle strength as it relates to reactive stepping performance. Specifically, testing muscle strength using handheld myometry has been shown to be a more sensitive measure for measuring higher degrees of strength for people with SCI (i.e., MMT scores > 4) (Noreau \& Vachon, 1998). The 
results of our study could also be limited by the participants' ability to anticipate when a release would occur. In order to avoid participants using anticipatory balance strategies, three 'sham' trials were included randomly during the testing session. However, if participants were able to anticipate a release, their subsequent step may not be indicative of a reactive response.

\subsection{Conclusions}

The mean of the spatial parameters measured using the Lean-and-Release test were found to have clinically useful test-retest reliability for the assessment of reactive stepping in people with iSCI, but the variability of the spatial parameters did not. The convergent validity of the mean step length, as measured using the Lean-and-Release test, was supported by significant correlations with measures of gait, balance, and leg strength. The findings suggest that the mean step length may be a particularly useful outcome to include in future assessments involving the Lean-and-Release test for the iSCI population. 


\section{References}

Berg, K., Wood-Dauphinee, S., \& Williams, J. I. (1995). The Balance Scale: reliability assessment with elderly residents and patients with an acute stroke. Scandinavian Journal of Rehabilitation Medicine, 27(1), 27-36.

Chan, K., Lee, J. W., Unger, J., Yoo, J., Masani, K., \& Musselman, K. E. (2020). Reactive stepping after a forward fall in people living with incomplete spinal cord injury or disease. Spinal Cord. 58, 185-193.

Chan, K., Unger, J., Lee, J. W., Johnston, G., Constand, M., Masani, K., \& Musselman, K. E. (2019). Quantifying balance control after spinal cord injury: Reliability and validity of the mini-BESTest. The Journal of Spinal Cord Medicine, 42(sup1), 141-148.

de Vet, H. C., Terwee, C. B., Knol, D. L., \& Bouter, L. M. (2006). When to use agreement versus reliability measures. Journal of Clinical Epidemiology, 59(10), 1033-1039.

Franchignoni, F., Horak, F., Godi, M., Nardone, A., \& Giordano, A. (2010). Using psychometric techniques to improve the Balance Evaluation Systems Test: The mini-BESTest. Journal of Rehabilitation Medicine, 42(4), 323-331.

Heffernan, M. G., Lee, J. W., Chan, K., Unger, J., Marzolini, S., Welsh, T. N., Masani, K., Musselman, K. E. (2021*) Spatial characteristics of reactive stepping among people living with chronic incomplete spinal cord injury. Spinal Cord, Manuscript submitted for review.

Hilliard, M. J., Martinez, K. M., Janssen, I., Edwards, B., Mille, M.-L., Zhang, Y., \& Rogers, M. W. (2008). Lateral balance factors predict future falls in community-living older adults. Archives of Physical Medicine and Rehabilitation, 89(9), 1708-1713. 
Hof, A. L., Gazendam, M. G., \& Sinke, W. E. (2005). The condition for dynamic stability. Journal of Biomechanics, 38(1), 1-8.

Hollman, J. H., Childs, K. B., McNeil, M. L., Mueller, A. C., Quilter, C. M., \& Youdas, J. W. (2010). Number of strides required for reliable measurements of pace, rhythm and variability parameters of gait during normal and dual task walking in older individuals. Gait \& Posture, 32(1), 23-28.

Hsiao-Wecksler, E. T., \& Robinovitch, S. N. (2007). The effect of step length on young and elderly women's ability to recover balance. Clinical Biomechanics, 22(5), 574-580.

Inness, E. L., Mansfield, A., Biasin, L., Brunton, K., Bayley, M., \& McIlroy, W. E. (2015). Clinical implementation of a reactive balance control assessment in a sub-acute stroke patient population using a 'lean-and-release' methodology. Gait Posture. 41(2), 529-34.

Jørgensen, V., Opheim, A., Halvarsson, A., Franzén, E., \& Roaldsen, K. S. (2017). Comparison of the Berg Balance Scale and the mini-BESTest for assessing balance in ambulatory people with spinal cord injury: Validation study. Physical Therapy, 97(6), 677-687.

Kendall, F. P., \& Al, E. (2010). Muscles: Testing and function with posture and pain. Lippincott Williams \& Wilkins.

Koo, T. K., \& Li, M. Y. (2016). A guideline of selecting and reporting intraclass correlation coefficients for reliability research. Journal of Chiropractic Medicine, 15(2), 155-163.

Lemay, J. F., Noamani, A., Unger, J., Houston, D. J., Rouhani, H., Musselman, K. E. (2020). Using wearable sensors to characterize gait after spinal cord injury: Evaluation of testretest reliability and construct validity. Spinal Cord, E-pub Oct 6. 
Maki, B. E., \& Mcilroy, W. E. (2006). Control of rapid limb movements for balance recovery: Age-related changes and implications for fall prevention. Age Ageing, 35(2), ii12-ii18.

Mansfield, A., Aqui, A., Danells, C. J., Knorr, S., Centen, A., DePaul, V. G., Schinkel-Ivy, A., Brooks, D., Inness, E. L., \& Mochizuki, G. (2018). Does perturbation-based balance training prevent falls among individuals with chronic stroke? A randomised controlled trial. BMJ Open, 8(8), e021510.

Mansfield, A., Inness, E. L., Komar, J., Biasin, L., Brunton, K., Lakhani, B., \& McIlroy, W. E. (2011). Training rapid stepping responses in an individual with stroke. Physical Therapy, 91(6), 958-969.

Mansfield, A., Inness, E. L., Lakhani, B., \& McIlroy, W. E. (2012). Determinants of limb preference for initiating compensatory stepping post- stroke. Archives of Physical Medicine and Rehabilitation, 93(7), 1179-1184.

Mansfield, A., Inness, E. L., Wong, J. S., Fraser, J. E., \& McIlroy, W. E. (2013). Is impaired control of reactive stepping related to falls during inpatient stroke rehabilitation? Neurorehabilitation and Neural Repair, 27(6), 526-533.

Martinez, K. M., Rogers, M. W., Blackinton, M. T., Cheng, M. S., \& Mille, M. L. (2019). Perturbation-induced stepping post-stroke: A pilot study demonstrating altered strategies of both legs. Frontiers in Neurology, 10, 711.

McCrum, C., Gerards, M. H. G., Karamanidis, K., Zijlstra, W., \& Meijer, K. (2017). A systematic review of gait perturbation paradigms for improving reactive stepping responses 
and falls risk among healthy older adults. European Review of Aging and Physical Activity, 14(1).

Medell, J. L., \& Alexander, N. B. (2000). A clinical measure of maximal and rapid stepping in older women. The Journals of Gerontology Series A: Biological Sciences and Medical Sciences, 55(8), M429-M433.

Mokkink, L. B., Terwee, C. B., Patrick, D. L., Alonso, J., Stratford, P. W., Knol, D. L., Bouter, L. M., \& de Vet, H. C. (2010). The COSMIN study reached international consensus on taxonomy, terminology, and definitions of measurement properties for health-related patient-reported outcomes. Journal of Clinical Epidemiology, 63(7), 737-745.

Mukaka M. M. (2012). Statistics corner: A guide to appropriate use of correlation coefficient in medical research. Malawi Medical Journal: The Journal of Medical Association of Malawi, 24(3), 69-71.

Noonan, V. K., Fingas, M., Farry, A., Baxter, D., Singh, A., Fehlings, M. G., \& Dvorak, M. F. (2012). Incidence and prevalence of spinal cord injury in Canada: A national perspective. Neuroepidemiology, 38(4), 219-226.

Noreau, L., \& Vachon, J. (1998). Comparison of three methods to assess muscular strength in individuals with spinal cord injury. Spinal cord, 36(10), 716-723.

Powell, L. E., \& Myers, A. M. (1995). The Activities-specific Balance Confidence (ABC) Scale. The Journals of Gerontology. Series A, Biological Sciences and Medical Sciences, 50A(1), M28-M34. 
Schwartz, S., Cohen, M. E., Herbison, G. J., \& Shah, A. (1992). Relationship between two measures of upper extremity strength: manual muscle test compared to hand-held myometry. Archives of physical medicine and rehabilitation, 73(11), 1063-1068.

Shah, G., Oates, A. R., Arora, T., Lanovaz, J. L., \& Musselman, K. E. (2017). Measuring balance confidence after spinal cord injury: the reliability and validity of the Activities-specific Balance Confidence Scale. The Journal of Spinal Cord Medicine, 40(6), 768-776.

Streiner, D. L., Norman, G. R., \& Cairney, J. (2015). Health measurement scales: A practical guide to their development and use. Oxford University Press.

Unger, J., Chan, K., Lee, J. W., Craven, B. C., Mansfield, A., Alavinia, M., Masani, K., \& Musselman, K. E. (2021). The effect of Perturbation-based Balance Training and Conventional Intensive Balance Training on reactive stepping ability in individuals with incomplete spinal cord injury or disease: A randomized clinical trial. Frontiers in Neurology, 12, 620367.

Unger, J., Chan, K., Scovil, C. Y., Craven, B. C., Mansfield, A., Masani, K., \& Musselman, K. E. (2019). Intensive balance training for adults with incomplete spinal cord injuries: Protocol for an assessor-blinded randomized clinical trial. Physical Therapy, 99(4), 420427.

Unger, J., Oates, A. R., Lanovaz, J., Chan, K., Lee, J. W., Theventhiran, P., Masani, K., \& Musselman, K. E. (2020). The measurement properties of the Lean-and-Release test in people with incomplete spinal cord injury or disease. The Journal of Spinal Cord Medicine, 1-10. 
Weaver, T. B. (2017). New insights into the landing phase of reactive stepping: Predictors of control, muscle recruitment, movement restraints and two-step responses. Doctoral dissertation, University of Waterloo, Waterloo Canada.

Werth, J., Bohm, S., Klenk, J., König, M., Sczuka, K. S., Schroll, A., Epro, G., Mandla-Liebsch, M., Rapp, K., Potthast, W., Arampatzis, A., \& Karamanidis, K. (2021). Stability recovery performance in adults over a wide age range: A multicentre reliability analysis using different lean-and-release test protocols. Journal of biomechanics, 125, 110584.

Wolfson, L. I., Whipple, R., Amerman, P., \& Kleinberg, A. (1986). Stressing the postural response. A quantitative method for testing balance. Journal of the American Geriatrics Society, 34(12), 845-850.

Yamaguchia, T., Masani, K. (2021*) Effects of aging on dynamic balance measures and their correlation during walking. Under Review. 


\section{Chapter 5}

\section{Discussion}

\subsection{Summary of Results}

The first objective of this study was to compare the spatial characteristics of the first reactive step between people with incomplete spinal cord injury (iSCI) and able-bodied (AB) individuals during the Lean-and-Release test. We hypothesized that, compared to AB individuals, people with iSCI would take first reactive steps that were shorter in length. However, the results presented in Chapter 3 did not support this hypothesis. Using independent t-tests, mean length, mean width, length variability, and width variability of the first reactive steps taken during the Lean-and-Release trials were compared between the two groups. The results of these comparisons demonstrated no significant differences in mean step length and mean step width, but significant differences between groups were found in step length variability and step width variability.

The second objective of this study was to assess the psychometric properties of the Lean-andRelease test for measuring the spatial parameters of reactive stepping for individuals with iSCI. We hypothesized the Lean-and-Release test would demonstrate a clinically useful degree of testretest reliability (intra-class correlation coefficients (ICC) $\geq 0.70$ ), as a measure of mean step length and mean step width, but not for step length variability and step width variability.

Additionally, we hypothesized that the means and variability of step length and step width would correlate with lower extremity strength and performance on other measures of balance and gait, indicating convergent validity of the Lean-and-Release test. The results presented in Chapter 4 indicated a clinically useful degree of test-retest reliability for mean step length and mean step 
width, but not for step length variability and step width variability. Convergent validity was demonstrated by significant moderately strong or strong correlations between mean step length and performance on other measures of balance and gait. Mean step length, step length variability, and step width variability were also shown to have significant moderately strong correlations with measures of lower extremity strength.

\subsection{Implications of Results}

An individual's ability to take a reactive step after an unexpected perturbation is one of the most vital components of postural control used to avoid falls (Maki \& McIlroy, 1996). However, despite its critical importance, trained therapists who assess balance in clinical populations do not prioritize the measurement of reactive balance (Sibley et al., 2011). The results presented in this study serve to further our understanding of the potential use of the Lean-and-Release test as a measure of reactive postural control for people with iSCI. First, this study demonstrated that step length variability and step width variability differed significantly between participants with iSCI and $\mathrm{AB}$ participants, suggesting these parameters can distinguish those with iSCI from those with more intact postural control. Second, this study has demonstrated that the mean step length and mean step width measurements of the Lean-and-Release test are reliable for clinical use. Third, certain spatial measures of the Lean-and-Release test (mean step length, step length variability, and step width variability) were shown to correlate with clinical measures of leg strength and balance, indicating performance on the test likely reflects postural control (Sibley et al., 2015). Together, these findings suggest that for people with iSCI, mean step length, step length variability and step width variability may be parameters of interest to clinicians using the Lean-and-Release test to assess reactive postural control. Future research examining the 
responsiveness or sensitivity to change of these spatial parameters, as measured with the Leanand-Release test, would provide greater insight into the clinical utility of this spatial parameter.

Although the results of this study support the clinical utility of the Lean-and-Release test for people with iSCI, certain limitations of the test must be considered. First, the instruments used to collect data during the Lean-and-Release test in this study are complex and expensive, which limits its accessibility in clinical environments. A more simplified version of the test has been used to measure the behavioural response (i.e. single step, multi-step, or fall) after a simulated forward fall in people with acquired brain injuries however, spatial parameters were not assessed (Borrelli et al., 2019). This simplified version of the Lean-and-Release test does not use a force transducer to precisely measure percentage of body weight supported by the horizontal cable before release, but instead a release is initiated manually by a therapist. This simplified method may be supported by research indicating the Lean-and-Release test is a reliable measure of spatial parameters of reactive stepping independent of lean angle (Werth et al., 2021). Second, the Lean-and-Release test may also be limited by a lack of correlation between its spatial measures and fall history. Specifically, the results in Chapter 3 of this study showed that compared to AB individuals, people with iSCI take first reactive steps that are more variable in length and width, however, no relationship was found between the variability of one's reactive step length or step width and their 3-month fall history. This finding indicates performance on the Lean-and-Release test may not be linked to one's propensity to fall. However, people with iSCI fall because of many unique factors that are not reflected in the Lean-and-Release test (Jørgensen \& Roaldsen, 2017; Singh et al., 2020) and 3-month fall history may have been subject to recall bias since it was a self-reported measure collected retrospectively. Finally, measures of 
step length and step width during the Lean-and-Release test may represent an oversimplified assessment of the spatial characteristics of an individual's reactive stepping response. Instead, assessing margin of stability (MoS) may provide a more comprehensive understanding of stepping performance during a dynamic situation such as the Lean-and-Release test (Hof et al., 2005). However, as outlined above, the setup required to measure certain parameters of the Leanand-Release test may be too complicated for widespread clinical use. Step length and step width are more accessible and easily attainable measures when compared to MoS.

Despite potential limitations of the Lean-and-Release test as a tool for clinical balance assessment, the results of our study may provide valuable information about reactive postural control of individuals after an iSCI. The results of Chapter 3 demonstrated a significant difference in reactive step length variability and step width variability between people with iSCI and $\mathrm{AB}$ individuals. These findings suggest that from one standardized simulated forward fall to the next, individuals with iSCI take a first reactive step more variable in length and width than $\mathrm{AB}$ individuals of similar age and sex. A similar study examining the onset of leg muscle activity during the Lean-and-Release test also found people with iSCI had increased variability from one trial to the next compared to AB counterparts (Chan et al., 2020). Additionally, during various walking conditions, individuals with iSCI have demonstrated increased variability in foot placement relative to centre of mass, MoS, step length, step width, and cadence (Day et al., 2011; Lemay et al., 2020). One potential explanation for the observed increase in variability of stepping for people with iSCI is the disruption in flow of sensory information caused by lesions of the spinal cord. Afferent information from the central nervous system is critical for maintaining balance and when the supply of this information is insufficient, error in the motor output is likely to increase (Dietz et al., 2002). In the context of this study, an individual's spinal cord lesion likely disrupts the flow of afferent information related to an unpredictable perturbation (the 
release of the cable) and increases the error of the motor output (the reactive step), thereby increasing the variability of step length and width from trial to trial. Increased variability of reactive step length and width may also be explained by impaired strength of the leg muscles. In chapter 4, a significant negative correlation was found between step length variability and strength of the ankle dorsiflexor muscles. Additionally, step width variability was found to have a significant negative correlation with the knee flexor muscles. These results suggest that people with iSCI who have weak knee flexor and ankle dorsiflexor muscles are likely to have increased variability of their reactive step length and step width. Previous studies examining the spatial parameters of gait in older adults have also suggested that a lack of muscle strength may contribute to increases in stepping errors and variability of step length and width (Bogen et al., 2020; Shin et al., 2012).

Results from this study may provide insight into potential targets for the training and rehabilitation of reactive stepping in people living with iSCI. Based on our findings, one potential goal of reactive balance training could be to reduce the variability of one's reactive step length and width. Repetitive practice is one training strategy that has shown promise for reducing the variability of gait parameters in people with iSCI (Wu et al., 2019) and therefore, could be an effective strategy for reducing variability in reactive stepping. Recently, an innovative approach to balance training that involved the principles of repetitive practice was tested in people with iSCI (Unger et al., 2021). This approach involved a trained therapist administering a series of repetitive perturbations to the participants and was shown to be feasible for use in ambulatory people with iSCI (Unger et al., 2021). Future research could examine the effectiveness of perturbation-based balance training for reducing the variability of reactive step length and width in people with iSCI. 
The results of chapter 4 demonstrated a relationship between strength of the leg muscles and reactive stepping performance. Therefore, strengthening the lower extremity muscles may be an appropriate target of balance training interventions. Although traditional methods of strength training could be useful, functional electrical stimulation (FES) may help to initiate stronger muscle contractions for people with iSCI. FES is applied through surface electrodes on the skin and generates artificial muscle contractions (de Freitas et al., 2018). An innovative reactive balance training approach combining FES of the lower leg and perturbation-based balance training was recently tested through a case study (Gauthier et al., 2020). The results demonstrated clinically significant improvements in measures of postural control (Gauthier et al., 2020) and thus, further study of this new method is warranted and could consider its effect on reducing variability of step length and width as well as improving strength of the leg muscles.

\subsection{Limitations}

A considerable limitation of this study was the size of the samples. For both included studies, twenty-one participants with iSCI met the criteria for inclusion. However, as a result of technical issues with the force plate and motion capture systems used to collect spatial data during the Lean-and-Release test, a number of data sets were lost or faulty and could not be used for further analysis. This demonstrates further the need for a more simplified and less technically complex version of the Lean-and-Release test so that clinicians can easily use the assessment tool and not lose valuable data.

Another limitation of this study was the underrepresentation of male participants. Data from three male and nine female participants were analyzed in the first study sample and the second study included two males and eight females. These proportions were not representative of the 
Canadian SCI population where males are more likely to have a SCI than females (Noonan et al., 2012) and therefore, the generalizability of our results may be limited.

A lack of data relating to the sensory function of our participants represents a potential limitation of this study. Specifically, one's sensory function is likely to impact the ability to determine body position during a given movement (i.e., proprioception). Proprioception is important for the precise control of movements like stepping (Qaiser et al., 2019), but is often impaired for individuals with SCI (Chisholm et al., 2016). Therefore, quantifying the varying degrees of sensory and proprioceptive function of our participants may have assisted with the interpretation of the study findings. Sensory function can be assessed by examining an individual's ability to sense position of a joint and cutaneous vibration (Gilman, 2002). These assessments have been used to quantify sensory function as it relates to balance for people with SCI (Arora et al., 2017).

Lastly, the experimental procedure may have been limited by the participant's propensity to anticipate when the cable supporting them would be released. In doing so, participants would have been using anticipatory balance strategies in addition to the reactive responses of interest. To minimize the participants' attempts to anticipate a release, 'sham' trials (i.e. no release occurs) were included randomly throughout the 10 true Lean-and-Release trials.

\subsection{Conclusions}

Compared with $\mathrm{AB}$ individuals, the length and width of the first reactive steps taken by people with iSCI are similar in magnitude but are significantly more variable. Future research evaluating balance training interventions for people with iSCI could evaluate whether reduced variability of step length and width are markers of improved postural control. Mean step length, and mean step width measured using the Lean-and-Release test, were found to be reliable measures of reactive 
postural control for people with iSCI. Additionally, the Lean-and-Release test had convergent validity for the measurements of mean step length, step length variability and step width variability in people with iSCI. However, a larger sample size is required to confirm these psychometric findings. 


\section{References}

Arora, T., Musselman, K. E., Lanovaz, J., \& Oates, A. (2017). Effect of haptic input on standing balance among individuals with incomplete spinal cord injury. Neuroscience letters, 642, 91-96.

Bogen, B., Moe-Nilssen, R., Aaslund, M. K., \& Ranhoff, A. H. (2020). Muscle strength as a predictor of gait variability after two years in community-living older adults. The Journal of Frailty \& Aging, 9(1), 23-29.

Borrelli, J. R., Junod, C. A., Inness, E. L., Jones, S., Mansfield, A., \& Maki, B. E. (2019). Clinical assessment of reactive balance control in acquired brain injury: A comparison of manual and cable release-from-lean assessment methods. Physiotherapy Research International, 24(4), e1787.

Chan, K., Lee, J. W., Unger, J., Yoo, J., Masani, K., \& Musselman, K. E. (2020). Reactive stepping after a forward fall in people living with incomplete spinal cord injury or disease. Spinal Cord. 58, 185-193.

Chisholm, A. E., Domingo, A., Jeyasurya, J., \& Lam, T. (2016). Quantification of Lower Extremity Kinesthesia Deficits Using a Robotic Exoskeleton in People With a Spinal Cord Injury. Neurorehabilitation and neural repair, 30(3), 199-208.

Day, K. V., Kautz, S. A., Wu, S. S., Suter, S. P., Behrman, A. L. (2011). Foot placement variability as a walking balance mechanism post-spinal cord injury. Clinical Biomechanics, 13,13 
de Freitas, G. R., Szpoganicz, C., \& Ilha, J. (2018). Does neuromuscular electrical stimulation therapy increase voluntary muscle strength after spinal cord injury? A systematic review. Topics in Spinal Cord Injury Rehabilitation, 24(1), 6-17.

Dietz, V., Müller, R., \& Colombo, G. (2002). Locomotor activity in spinal man: Significance of afferent input from joint and load receptors. Brain: A Journal of Neurology, 125(Pt 12), $2626-2634$.

Gauthier, C., Chan, K., Masani, K., Musselman, K. E. (2020). Perturbation-based training in combination with functional electrical stimulation: A promising mixed-methods case study. Archives of Physical Medicine and Rehabilitation, 101(11): (abstract e26).

Gilman S. (2002). Joint position sense and vibration sense: anatomical organisation and assessment. Journal of neurology, neurosurgery, and psychiatry, 73(5), 473-477.

Hof, A. L., Gazendam, M. G., \& Sinke, W. E. (2005). The condition for dynamic stability. Journal of biomechanics, 38(1), 1-8.

Jørgensen, V., \& Roaldsen, K. S. (2017). Negotiating identity and self-image: perceptions of falls in ambulatory individuals with spinal cord injury - a qualitative study. Clinical Rehabilitation, 31(4), 544-554.

Lemay, J. F., Noamani, A., Unger, J., Houston, D. J., Rouhani, H., Musselman, K. E. (2020). Using wearable sensors to characterize gait after spinal cord injury: Evaluation of testretest reliability and construct validity. Spinal Cord, E-pub Oct 6.

Maki, B. E., \& McIlroy, W. E. (1996). Postural control in the older adult. Clinics in Geriatric Medicine, 12(4), 635-658. 
Noonan, V. K., Fingas, M., Farry, A., Baxter, D., Singh, A., Fehlings, M. G., \& Dvorak, M. F. (2012). Incidence and prevalence of spinal cord injury in Canada: A national perspective. Neuroepidemiology, 38(4), 219-226.

Qaiser, T., Eginyan, G., Chan, F., \& Lam, T. (2019). The sensorimotor effects of a lower limb proprioception training intervention in individuals with a spinal cord injury. Journal of neurophysiology, 122(6), 2364-2371.

Shin, S., Valentine, R. J., Evans, E. M., \& Sosnoff, J. J. (2012). Lower extremity muscle quality and gait variability in older adults. Age and Ageing, 41(5), 595-599.

Sibley, K. M., Beauchamp, M. K., Van Ooteghem, K., Straus, S. E., \& Jaglal, S. B. (2015). Using the systems framework for postural control to analyze the components of balance evaluated in standardized balance measures: a scoping review. Archives of Physical Medicine and Rehabilitation, 96(1), 122-132.

Sibley, K. M., Straus, S. E., Inness, E. L., Salbach, N. M., \& Jaglal, S. B. (2011). Balance assessment practices and use of standardized balance measures among Ontario physical therapists. Physical Therapy, 91(11), 1583-1591.

Singh, H., Shibi Rosen, A., Bostick, G., Kaiser, A., \& Musselman, K. E. (2020). Exploring the causes and impacts of falls among ambulators with spinal cord injury using photovoice: A mixed methods study. BMJ Open. 10, e039763.

Unger, J., Chan, K., Lee, J. W., Craven, B. C., Mansfield, A., Alavinia, M., Masani, K., \& Musselman, K. E. (2021). The effect of Perturbation-based Balance Training and Conventional Intensive Balance Training on reactive stepping ability in individuals with 
incomplete spinal cord injury or disease: A randomized clinical trial. Frontiers in Neurology, 12, 620367.

Werth, J., Bohm, S., Klenk, J., König, M., Sczuka, K. S., Schroll, A., Epro, G., Mandla-Liebsch, M., Rapp, K., Potthast, W., Arampatzis, A., \& Karamanidis, K. (2021). Stability recovery performance in adults over a wide age range: A multicentre reliability analysis using different lean-and-release test protocols. Journal of biomechanics, 125, 110584.

Wu, M. M., Brown, G. L., Kim, K. A., Kim, J., \& Gordon, K. E. (2019). Gait variability following abrupt removal of external stabilization decreases with practice in incomplete spinal cord injury but increases in non-impaired individuals. Journal of Neuroengineering and Rehabilitation, 16(1), 4. 


\section{Copyright Acknowledgements}

RightsLink Primtable License

SPRINGER NATURE LICENSE

TERMS AND CONDITIONS

Sep 21,2021

This Agreement between University of Toronto -- Matthew Heffernan ("You") and Springer Nature ("Springer Nature") consists of your license details and the terms and conditions provided by Springer Nature and Copyright Clearance Center.

License Number

5126621213709

License date

Aug 12, 2021

Licensed Content

Publisher

Springer Nature

Licensed Content

Publication

Spinal Cord

Licensed Content Title

Reactive stepping after a forward fall in people living with incomplete spinal cord injury or disease

Licensed Content Author Katherine Chan et al

Licensed Content Date Jul 29, 2019

Type of Use Thesis/Dissertation

Requestor type academic/university or research institute

Format print and electronic

Portion figures/tables/illustrations

Number of 1

figures/tables/illustrations 
Author of this Springer

Nature content

no

Title

Spatial characteristics of reactive stepping among people living with incomplete spinal cord injury

Institution name

University of Toronto

Expected presentation

date

Sep 2021

Portions

Figure 1 